Approved for public release. Distribution is unlimited.

\title{
NETWORK TECHNOLOGY FOR DEPOT
} MODERNIZATION

C. J. Hostick

December 1990

P apared for the U.S. Department of the Army under a Related Services Agreement with the U.S. Department of Energy under Contract DE-AC06-76RLO 1830

Pacific Northwest Laboratory

Richland, Washington 99352 


\section{PREFACE}

This report was prepared by the Pacific Northwest Laboratory to summarize existing and emerging information system technology and standards applicable to Depot System Command (DESCOM) modernization efforts. The intent of this summarization is to provide the REvitalization of Army Depots for the Year 2000 (READY 2000) team a clear understanding of the enabling information system technologies required to support effective modernization activities. The success of this attempt can be measured by 1) the ability of the READY 2000 team to understand the modernization strategies and architecture of effective information systems, and 2) the identification by the READY 2000 team of the central role an information system plays in integrating the industrial enterprise.

Much of the information contained in this report was acquired during the last year in support of the U.S. Army Armament, Munitions, and Chemical Command (AMCCOM) Facility Integrated Manufacturing Management System (FIMMS) project at PNL, which is targeting the modernization of plant-wide information systems at Army Ammunition Plants. The AMCCOM FIMMS initiative provides a good precedent for DESCOM's information system modernization and will continue to provide invaluable information concerning the modernization of U.S. Army production and support facilities. 


\section{EXECUTIVE SUMMARY}

The objective of information system rnodernization is to improve the effectiveness of an organization in performing its mission. Information system modernization strives to meet this objective by creating an environment where data is electronically captured near the source and readily available to all areas of the organization. Advanced networks, together with related information system technology, are the enabling mechanisms that make modern information system infrastructures possible.

The intent of this paper is to present an overview of advanced information system network technology to support depot modernization planners in making technology management decisions. Existing and emerging Open System Interconnection (OSI) and Government Open System Interconnection Profile (GOSIP) standards are explained, as well as a brief assessment of existing products compliant with these standards.

Finally, recommendations for achieving plant-wide integration using existing products are presented, and migration strategies for full OSI compliance are introduced. 


\section{CONTENTS}

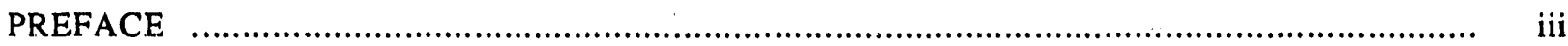

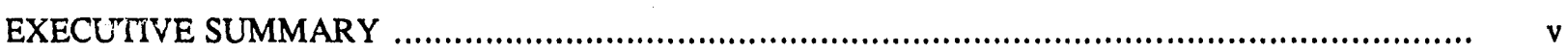

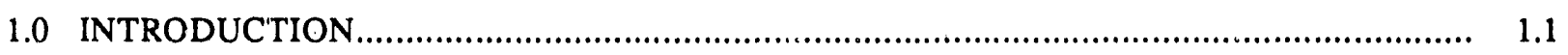

1.1 CONNECTION BETWEEN INFORMATION SYSTEMS AND TOTAL QUALITY MANAGEMENT.

1.2 COMMON MISCONCEPTIONS REGARDING INFORMATION SYSTEM TECHNOLOGY

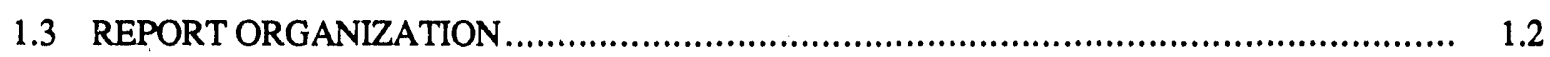

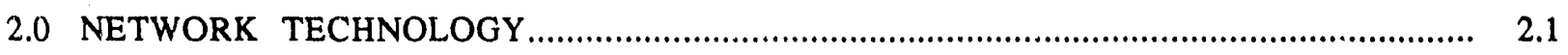

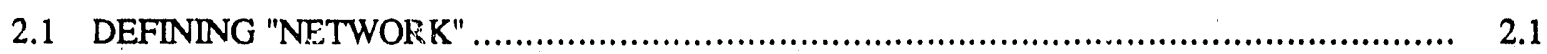

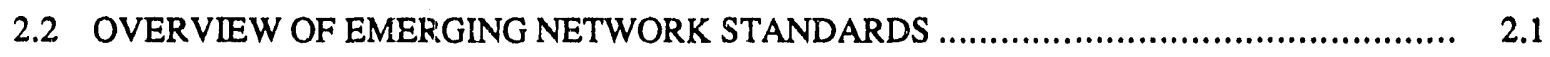

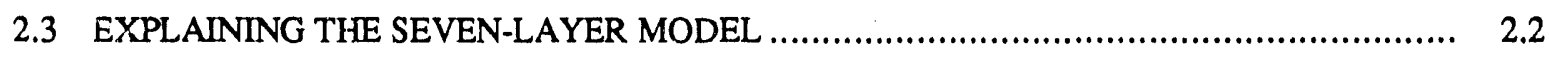

2.4 GOSIP: THE FEDERAL GOVERNMENT AND OSI ........................................... 2.3

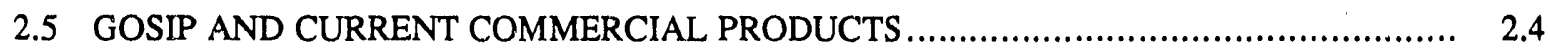

2.6 NETWORK COMPONENTS AND RELATED TECHNOLOGY ............................... 2.5

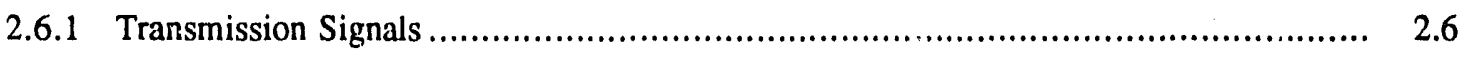

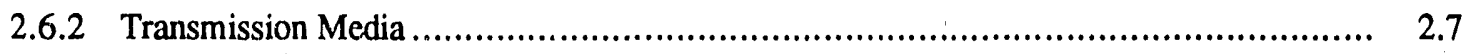

2.6.3 Network Components ........................................................................... 2.11

2.6.4 Related Information System Terminology................................................... 2.11

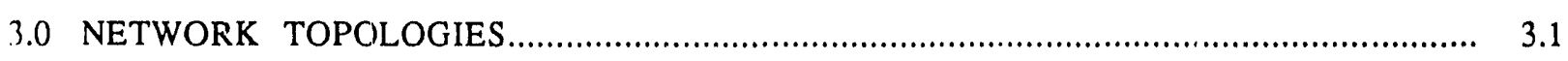

3.1 OVERVIEW OF NETWORK CONFIGURATIONS................................................ 3.1

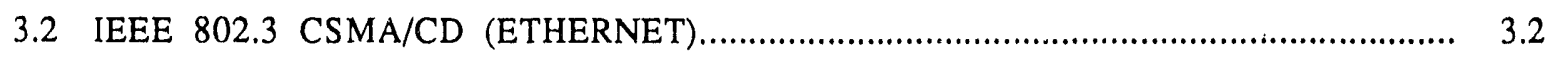

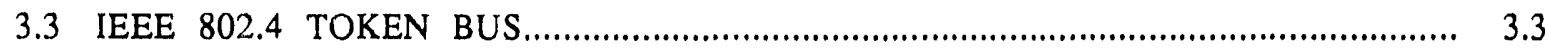




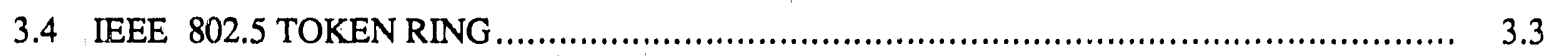

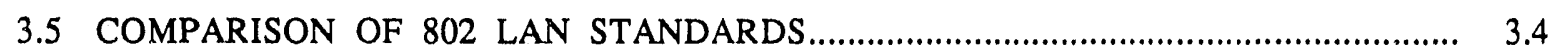

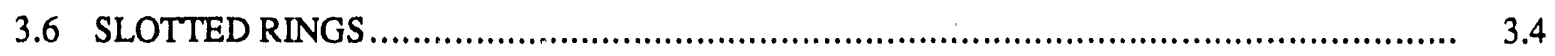

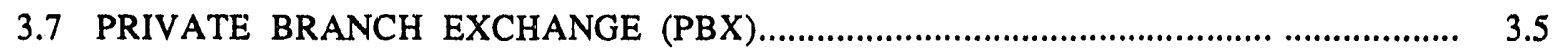

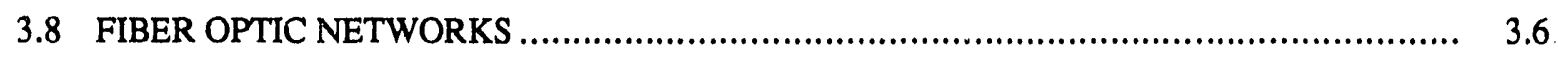

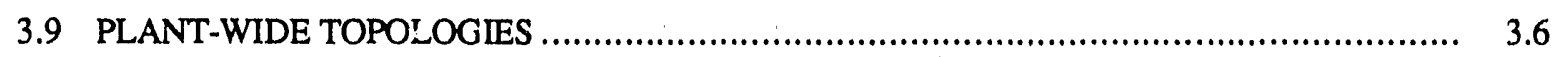

4.0 NETWORK MIGRATION STRATEGIES ........................................................... 4.1

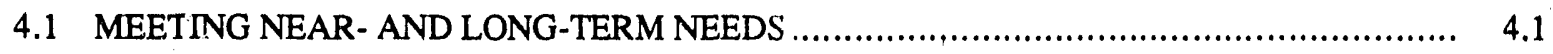

4.2 PHASING IN OSI-COMPLIANT TECHNOLOGY ............................................. 4.1

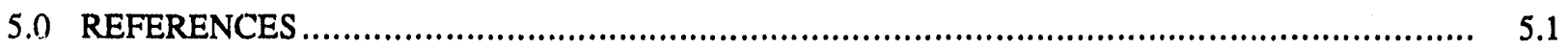




\section{FIGURES}

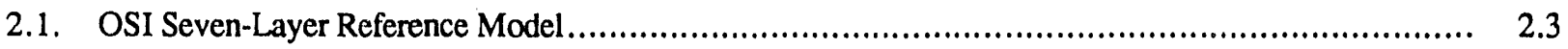

2.2. Existing Communication Architectures Versus the OSI Model................................................. 2.5

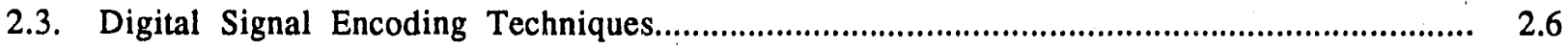

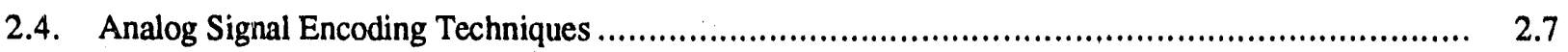

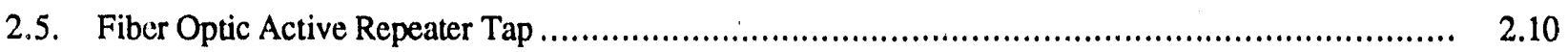

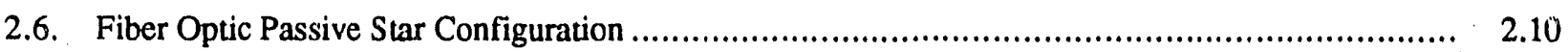

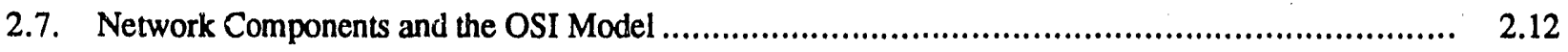

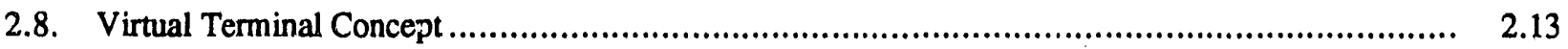

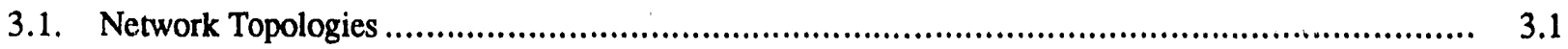

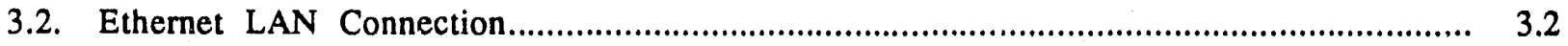

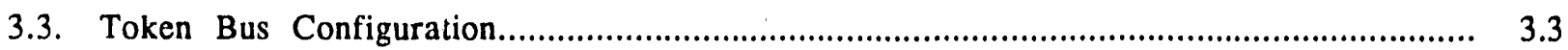

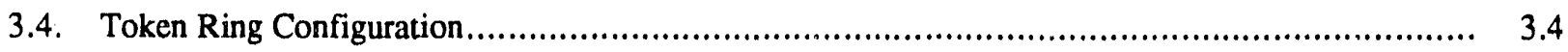

3.5. Wire Center for Improved Ring Reliability ......................................................................

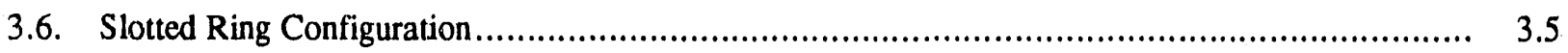

3.7. Communication System Using Private Branch Exchanges................................................... 3.6

3.8. Ring Configuration Using Fiber Distributed Data Interface.................................................... $\quad 3.7$

3.9. FDDI Ring Used as a Backbone to Connect LANs and Computers............................................... 3.7 


\subsection{INTRODUCTION}

\subsection{CONNECTION BETWEEN INFOR- MATION SYSTEMS AND TOTAL QUAL- ITY MANAGEMENT}

Information generation and transfer within manufacturing environments is like material handling: if you have to do it, you want to do it as efficiently as possible. As initiatives such as cost reductions and Total Quality Management (TQM) gain momentum, the amount of information that must be generated and transferred will continue to increase dramatically. Instead of monthly reports regarding quality and cost, managers will require daily and weekly information regarding the status of their operations to meet increasingly difficult performance objectives. Moving this information efficiently and on time will require an information system infrastructure that does not currently exist in the U.S Army Depot environment.

Information system modernization strives for an environment where data is electronically captured near the source and is readily accessible to all areas of the organization. Information exchange between plant information systems is transparent to the user; i.e., although multiple computer systems, software applications, and transmission media may exist onsite, the system appears to the user to be one integrated system directly accessible from his or her work location.

Advanced networks, together with related information systern technology, are the enabling mechanisms that make modem information system infrastructures possible. Unfortunately, most modernization planners have little familiarity with network technology and how it is applied. Planners are faced with an avalanche of terms (usually presented in acronym form), volumes of literature, constantly evolving software/hardware products, and emerging government communication standards. While this information may be suitable for technical experts in computer communications, modemization planners must work at a higher level of systems planning, which requires an understanding of the functionality, if not the specifics, of a broad range of manufacturing technology. The intent of this paper is to present a systems view of network technology for depot modernization planners and to provide sufficient overview to assist planners in their near-term information system modernization efforts.

\subsection{COMMON MISCONCEPTIONS RE- GARDING INFORMATION SYSTEM TECHNOLOGY}

A lack of understanding regarding the state of information system technology presents another barrier to effective modemization planning. So much literature has been produced regarding information system products and advancements that the casual reader is inclined to think that 1) communication standards are well supported by commercial products, and/or 2) nearly all major non-government U.S. manufacturing facilities have some type of plant-wide communication system installed.

Communication standards compatible with emerging government requirements principally consist of the Open Systems Interconnection (OSI) standards for data exchange, as sanctioned by the International Standards Organization (ISO). These standards have been adnpted by such firms as General Motors and Boeing, which have developed and supported products such as Manufacturing Automation Protocol (MAP) and Technici and Office Protocol (TOP). A review of the vast amous.s of MAP/TOP literature would suggest that these OSI-compliant products must have a significant and ever increasing installed user base, suggesting in turn that modernization planners have a wide range of MAP/TOP-compatible products and vendors from which to choose. Were this actually the case, information system modernization planning would be a simple task, as depots could be immediately compliant with OSI standards simply by purchasing MAP/TOP communication products. The reality of MAP/TOP, however, is strikingly different from what the literature would suggest. As of 1988 , there were only approximately $75 \mathrm{MAP}$ installations and $6 \mathrm{TOP}$ installations world wide (Bernard 1988). While this user base is increasing, it is not yet sufficiently large to support a wide variety of MAP/TOP compatibleproducts nor a wide vendor base. The, result is that depot modernization planners can choose MAP/TOP to modemize their information systems, but they will have less effective systems that will cost more money than a variety of proven and competing modemization strategies that can also lead to full OSI compliance.

Another belicf is that current depot information systems lag significantly behind private industry. While it is true that lcading manufacturing firms do 
have plant-wide integrated information systems in place, the majority of private installations do not. A survey (Russell 1988) of 1800 industrial sites with more than 100 employees resulted in in-depth statistics regarding 573 facilities. Less than 25 percent of these sites had a plant-wide communication system in place, and only 40 percent planned to have a network installed by 1990 . Broadband and twisted-pair media accounted for nearly 70 percent of all installed network systems. Communication protocols planned for nearterm networks include a broad range of options. Ethernet $^{\text {TM }}$-based protocols are the most common, accounting for over 30 percent of planned network systems. IBM's\$ proprietary SNA protocols are planned for 21 percent of near-term networks.

Finally, in planning for modernized depot information systems, it is necessary to review the network design features viewed as most important by private installations. The overall ranking of features viewed as most important are as follows (Russell 1988):

1. network reliability
2. compatibility with existing equipment

3. service responsiveness

4. total cost of the network as a whole

5. speed of the network

6. rarge of devices that can be connected.

\subsection{REPORT ORGANIZATION}

The building blocks for effective information systems are presented in the following chapters. An overview of network standards and components is presented in Chapter 2. Network topologies are presented in Chapter 3, and network migration strategies for achieving compliance with emerging standards are presented in Chapter 4.
Ethernet $^{\mathrm{TM}}$ is a trademark of Xerox Corporation, Stamford, Connecticut.

$\mathrm{IBM}^{(3)}$ is a registered trademark of International Business Machines, Inc., Armonk, New York. 


\subsection{NETWORK TECHNOLOGY}

\subsection{DEFINING "NETWORK"}

The term "network" refers to a collection of physical nodes and transmission paths for processing and distributing information. A "network architecture" refers to the design philosophy that integrates hardware and software components with a set of layers and protocols to operate and manage the network.

A network and its corresponding architecture provides the con munications links between individuals on the job and the balance of the plant's information system resources. An adequate network anchitecture is key to a successful information system, as it serves to integrate an organization by allowing for the dissemination of performance data. This chapter reviews the standards for network architecture being developed by the federal govemment and existing/emerging network technologies. The purpose of this review is to identify the network technologies of choice for modernization activities during the next several years, and expected network technologies that will be required through the 1990s.

\subsection{OVERVIEW OF EMERGING NET. WORK STANDARDS}

Twenty years ago little need for network standards existed; what computing resources there were were primarily mainframes running batch application programs, with minimal terminal feeds. Information systems were oriented towards computing processes and not data communication. The International Organization for Standardization (ISO) and the International Telegraph and Telephone Consultative Committee (CCITT) organized study groups in the 1960 s to investigate data ccmmunication standards and the use of phone lines for data transmission. Standards regarding cable connectionis were established, and standards for various other aspects of network communication were pursued as the use of network technology expandied in the 1970s. It soon became apparent that multiple organizations were producing standard specifications for network communications without a common "master plan" that would ensure compatibility once all specifications were complete. In 1978 ISO initiated an effort to develop a reference model for data communication that would serve as a basis for all future development of data communication standards. Once completed, exisling standards were to be adapted to the new reference model, and areas where no standards existed were to be identified and developed.

A standard reference Open System Interconnection (OSI) model was completed and approved by ISO in May of 1983 and accepted with primarily editorial changes by CCITT (CCITT X.200).

The term "open" in the OSI model signifies one of the primary objectives of the standard, which was to enable products from multiple vendors that are OSI compliant to be easily integrated and combined into effective network communication systems. This was accomplished by dividing the process of network communication into seven layers, with a clear definition of interface requirements or protocols between each layer. Thus, each OSI layer defines the protocols for communication products providing services at that level. This seven-layer approach effectively defined "building blocks" that could be used to construct a network communication system. This approach is also a hedge against future advancements in communication technology, because future changes can be isolated to some subset of the total OSI reference model. This approach minimizes the fraction of the standard rendered obsolete by technology change at any one time.

The OSI seven layers are as follows:

\section{Application Layer}

The application layer selects the appropriate overall network service for the user's software application. Electronic mail and file transfer capabilities are examples of application layer services.

\section{Presentation Layer}

The presentation layer resolves syntax differences and performs data reformatting of the user's application.

\section{Session Layer}

The session layer coordinates interaction between user application processes on the different nosts. This layer serves to bind and unbind the different hosts into a communicating relationship. 


\section{Transport Layer}

The transport layer provides transparent transfer of data between session entities or hosts. Users are provided with the means to establish, maintain, and release transport connections.

\section{Network Layer}

The network layer provides the switching and routing functions needed to establish, maintain, and terminate switched connections.

\section{Data Link Layer}

The data link layer provides the functional and procedural means to establish, maintain, and release data link connections among network entities. For example, the method to determine when a particular terminal can have access to the network is specified by this level.

The data link layer is split into two sublayers. The lower sublayer is referred to as the Medium Access Control (MAC) sublayer, which has three variants in the IEEE 802 LAN standards adopted by OSI. The upper sublayer, which provides a uniform interface to the network layer is the same for all three variants. The OSI-compliant upper data link sublayer is referred to as IEEE 802.2 or Logical Link Control (LLC).

\section{Physical Layer}

The physical layer provides the physical medium for the information flow. It defines the mechanical and electrical interfaces to the network and the bit level data flow. The type of information included in physical layer interface standards consists of the number of volts to use for bit representation, the amount of time a bit occupies, data rates, etc.

\subsection{EXPLAINING THE SEVEN-LAYER MODEL}

These seven layers of the OSI reference model $c^{\prime} \wedge n$ be charecterized as three top levels that directly support the software application prosess, three bottom layers concerned with the transriussion of data over the physical media (e.g., wire), and the transport layer that completes the data transmission.
Network communications within the seven-layer reference model concept is illustrated by Figure 2.1.

One computer program contains some information to be passed to another program existing at some node on the network. The program gives the data (i.e., bit stream) to the application layer. The application layer performs a variety of functions. For example, to deal with a network with incompatible terminal types, the application layer defines an abstract network virtual terminal. Each terminal is handled by a piece of software that maps the functions of the real terminal onto the network virtual terminal. In this thanner, a location on one type of terminal screen can be mapped to another incompatible terminal type.

Another feature performed by the application layer is file transfer. Because different file systems have different file-naming conventions and different ways of representing text lines, these differences must be accounted for before data is passed to the presentation layer.

The presentation layer handles syntax differences in the data (e.g. ASCII versus EBCDIC) by converting the data into a standard encoding before passing the data to the session layer. The session layer is concerned with data traffic control and synchronization between sender and receiver. The transport layer, which accepts data from the session layer, splits up the data into smaller units and passes the units or packets to the network layer. The network layer determines how packets are routed from source to destination and controls network bottlenecks. Data is then passed to the data link layer and onto the physical layer.

The physical layer transmits raw bits over the communication channel. It determines such issues as how many volts should be used to represent a 1 or a 0 , and how many microseconds a bit lasts. The signal is then transmitted over the physical media to the physical layer of the receiving node, where the process is reversed. The raw signal is passed to the data link layer, : vhich ensures that the signal is free from errors. This tas is performed by having the sender break the input data up into frames consisting of a few hundred bytes, transmit the frames sequentially, and process the acknowledgem $\mathrm{nt}$ frames sent back by the receiver. Error-free data frames are passed up to the network layei and continue up through to the receiver's application layer.

A full implementation of the OSI layer stack at the work station level would require excessive main 


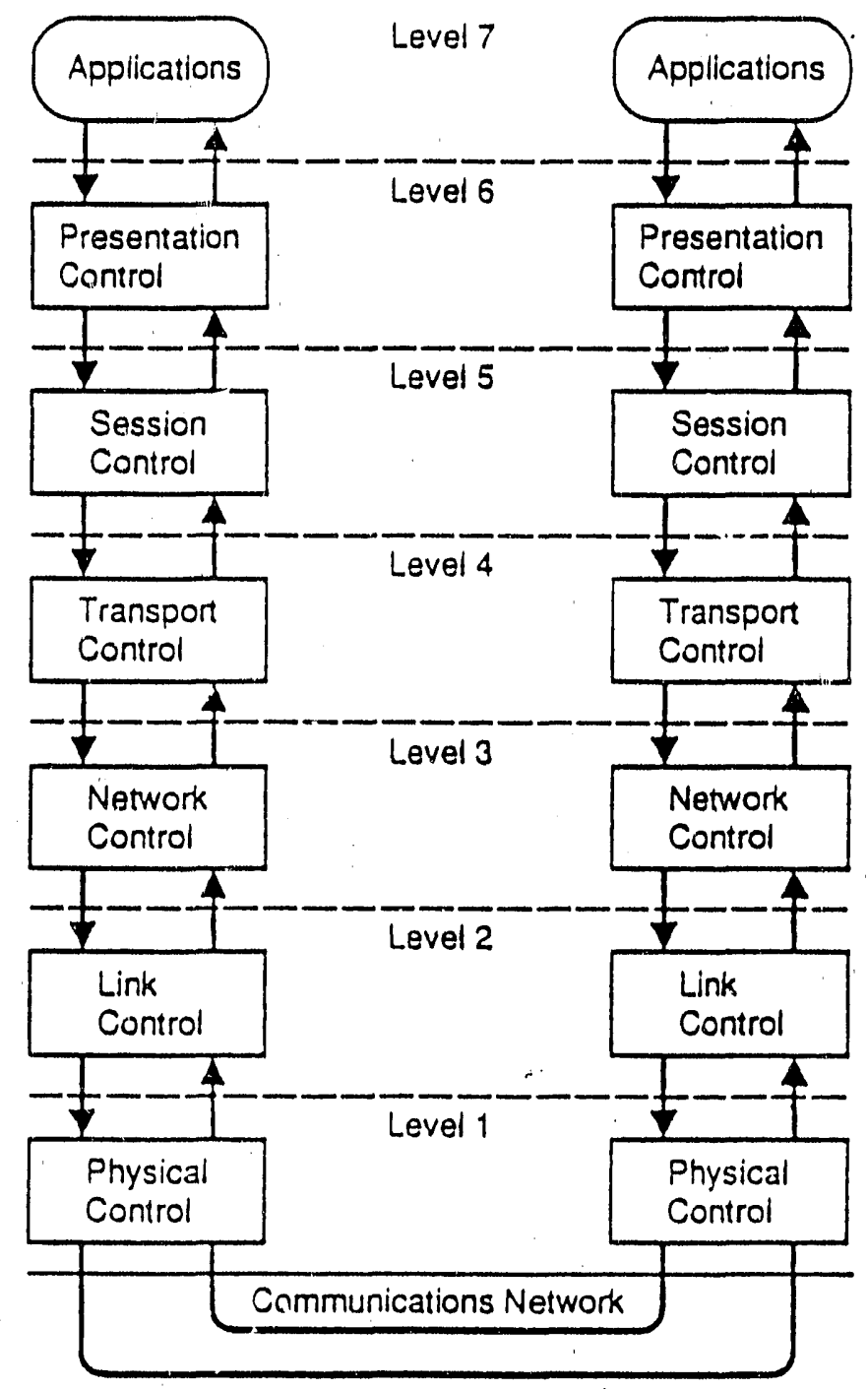

Figure 2.1. OSI Seven-Layer Reference Model

memory or expensive communication cards. One technique for alleviating this problem is to distribute the communication stack so that the session and lower layers are resident on a shared file server, leaving only the presentation/application layers on the work stations. The link between the work station applications and the server stack is provided by a remote procedure call mechanism.

\subsection{GOSIP: THE FEDERAL GOVERN. MENT AND OSI}

In August of 1988, the federal government published the Federal Information Processing Standards (FIPS) Publication 146, adopting the OSI reference model for data comrnunication protocols. This standard, referred to as the Government Open System
Interconnection Profile (GOSIP), has the following objectives:

- to achieve interconnection and interoperability of computers and systems that are acquired from different manufacturers in an open system environment

- to reduce the costs of computer network systems by increasing altranative sources of supply

- to facilitate the use of advanced technology by the federal government

- to stimulate the development of commercial products compatible with OSI. 
FIPS 146 (i.e., GOSIP) is not yet a complete standard. Full specification of data communication protocols compliant with the OSI reference model is not expected until after 1994. The purpose of the 1988 FIPS 146 release and later interim standards, however, is to slow the continued expansion of proprietary, non-interoperable vendor network communication products within the federal government until full OSI compatibility can be supported in the marketplace. This is being accomplished by implementing GOSIP standards that gradually migrate towards full OSI reference model compliance. For example, the first version of GOSIP (FIPS 146) only specifies network communication protocols for OSI layers $1,2,3$ and 7. These specifications include the following:

\section{- Layer 7--File Transfer, Ac ess and Management} (FTAM)

\section{- Layer 3--X.25 for Wide Area Networks}

- Layer 2,1-CSMA/CD (IEEE 802.3), Token Bus (IEEE 802.4), Token Ring (IEEE 802.5).

These specifications will be expanded within each layer, and new layers will be covered in future GOSIP releases. For example, the physical and data link layers for fiber optic local area networks, as implemented by the Fiber Distributed Data Interface (FDDI) conceph, is expected to be included in the next GOSIP version due in 1990.

Eventually, compliance with GOSIP standards for new hardware/software procurements for Government facilities will be required 18 months after each GOSIP standard is released. However, at the time of this writing, compliance is not mandatory until validation testing for computer product and services is fully established.

\subsection{GOSIP AND CURRENT COMMER- CIAL PRODUCTS}

To understand the gradual migration strategy of GOSIP requires understanding of the commercial products that exist today. I't a broad range of economical and effective OSI-compliant product and services were readily available, GOSIP/OSI compliance would have been fully specified by FIPS 146 . The reality is that there is a broad spectrum of commercial products in the market place capable of providing the functionality of the OSI seven layers, and with the exception of such architectures as MAP and TOP, these products may not be OSI compliant. This is illustrated by
Figure 2.2, showing the diversity of some of the commercially available architectures.

Three classes of emerging network architectures that are battling for the bulk of the data communication market place are OSI, SNA, and TCP/IP.

Products with OSI compatibility are widespread for the data link and physical protocol layers, because the OSI reference model includes a broad range of acceptable protocols at these levels. Commercial products that encompass all seven of the OSI levels are best represented by General Motor Corporation's Manufacturing Automation Protocol (MAP), and Boeing Computer Services Technical and Office Protocol (TOP). Although defined b: General Motors and Boeing, MAP and TOP softwa' is produced by vendors such as Retix Internetworkung Co. and Touch Communications Inc. While MAP and TOP are currently the leading network products that are OSI compliant, they have two major shortcomings that significantly inhibit their acceptance into the market place:

- Lack of an installed base has minimized the development of compatible software applications.

- Networking cards for MAP/TOP cost $\$ 2,000$ to $\$ 7,000$ each, more than three times thie cost of comparable cards for competing network architectures.

As additional vendors support the OSI reference model, and as the cost of interface cards drop, OSIcompatible products will eventually secure a substantial portion of the network communications market. Meanwhile, however, OSI-compatible products are not sufficiently mature to justify full OSI/GOSIP compliance within the federal government.

Systems Network Architecture (SNA), an IBM Corporation proprietary protocol suite, is a de facto industry standard due to IBM's control of $80 \%$ of the mainframe market. SiNA is implemented at over 25,000 sites and is an c zonomical and mature technology. IBM has faced a dilemma in recent years: providing network products to help customers network nonIBM machines with IBM equipment using non-proprietary standards will help the integration needs of customers, but may result in lost sales. IBM's apparent solution is to remain firm in supporting SNA as the protocol of choice for IBM systems while pursuing OSI products to network multivendor hardware environments into an SNA environment. In other words, IBiA products will be networker using SNA, and products from other vendors will use OSI protocols, which 


\begin{tabular}{|c|c|c|c|c|c|c|c|}
\hline Layer & $\begin{array}{l}\text { OS } \\
\text { Model }\end{array}$ & Map & Top & LAN & $\begin{array}{l}\text { IBM } \\
\text { SNA }\end{array}$ & $\begin{array}{l}\text { DEC } \\
\text { DNA }\end{array}$ & ARPANET \\
\hline 7 & Application & $\begin{array}{l}\text { MMS } \\
\text { FTAM }\end{array}$ & $\begin{array}{l}X .400 \\
\text { FTAM }\end{array}$ & Server & LU6.2 & OSAK & $\begin{array}{l}\text { TELNET } \\
\text { FTP }\end{array}$ \\
\hline $\begin{array}{l}6 \\
5\end{array}$ & $\begin{array}{l}\text { Presentation } \\
\text { Session }\end{array}$ & & & \multirow{3}{*}{$\begin{array}{l}\text { NET. } \\
\text { BIOS }\end{array}$} & VTAM & & \\
\hline 4 & Transport & & & & \multirow[t]{2}{*}{ NCP } & & TCP \\
\hline 3 & Network & & & & & & IP \\
\hline 2 & $\begin{array}{l}\text { Data } \\
\text { Link } \\
\text { Control }\end{array}$ & $\begin{array}{l}\text { Token } \\
\text { Bus } \\
\text { 8C2.4 }\end{array}$ & $\begin{array}{l}\text { Ether } \\
\text { net } \\
802.3\end{array}$ & $\begin{array}{c}\text { Token } \\
\text { Ring } \\
802.5 \\
\text { Also } \\
802.3 \text {, } \\
302.4\end{array}$ & SDLC & 802.3 & $\begin{array}{l}\text { Many } \\
\text { options }\end{array}$ \\
\hline 1 & Physical & $\begin{array}{l}\text { Broad- } \\
\text { band }\end{array}$ & $\begin{array}{l}\text { Base- } \\
\text { hand }\end{array}$ & $\begin{array}{l}\text { Broad } \\
\text { or } \\
\text { Base- } \\
\text { band }\end{array}$ & & $\begin{array}{l}\text { Broad } \\
\text { or } \\
\text { Base- } \\
\text { band }\end{array}$ & \\
\hline
\end{tabular}

Figure 2.2. Existing Communication Architectures Versus the OSI Miodel

will feed a gateway to the SNA communication sys." tem. In this manner, end-users rely on both OSI and SNA for their communication network. IBM has developed and is currently selling OSI-compliant products in Europe while monitoring development of the U.S. market.

Transmission Control Protocol/Internet Protocol (TCP/IP), a series of specifications originally developed in support of U.S. Department of Defense networks, is the world's most widely used set of non-proprietary network standards. TCP/IP is a network standard supported by more than a hundred vendors which, due to the slowness of OSI product availability, has become a de facto standard for integrating multivendor systems. As a non-proprietary solution for integrating multivendor systems, TCP/IP fulfills most of the objectives stated for GOSIP in FIPS 146. Until such time as OSI products achieve the capability and cost effectiveness of TCP/IP, it is expected that TCP/IP will be the protocol of choice for computer network architectures.
TCP/IP vendors are also pursuing the development of TCP/IP to OSI gateway products (GOVT Computing News 1989). In effect, these products allow TCP/IP protocols to serve as a migration strategy to GOSIP compliance. This strategy allows the use of readily availahle TCP/IP products to meet GOSIP objectives in the near-term while facilitating the eventual integration of OSI standards.

\subsection{NETWORK COMPONENTS AND RE- LATED TERMINOLOGY}

An understanding of common network terminology is essential for understanding the basics of network systems. A complex material handling system is composed of an assortment of system "building blocks" (e.g., conveyors, lift tables, hoppers, etc.); likewise, network systems consist of similar building blocks. An overview of network components and related terminology is presented in the following section 3 . 


\subsubsection{Transmission Signals}

The function of computer networks is to allow binary data streams transmiti d from one node (i.e., terminal or computer) to be received and correctly interpreted by another node. Bit stream transmission signals can be classified into two $\mathrm{g}$ ineral categories, digital transmission and analog transmission.

Digital transmission uses constant voltage levels (or light equivalent for fiber optics) to represent 0 and 1 bits during transmission. Three different encoding iechniques are illustrated by Figure 2.3. Straight binary encoding, sometimes used for coaxial cable, simply assigns a non-zero voltage for a 1 bit and 0 volts for a 0 bit. One drawback of straight binary signaling is that the receiver has no way of determining when each bit starts and ends. A technique referred to as Manchester encoding solves this problem by dividing each bit period into two equal intervals. A 1 bit is sent on high voltage during the first interval and low in the second (reverse for $0 \mathrm{bit}$ ). A variation of this technique is referred to as differential Manchester encoding, where a 1 bit is indicated by the absence of a transition at the start of the interval. The differential scheme requires somewhat more complex equipment but offers better noise immunity.

Advan.ages of digital signaling are numerous. As computers are digital based, interfaces betwecn computers and digital transmission on coaxial cable (i.e., baseband) are cheaper because no signal modulation is required. Digital transmissions also have the potential for very low error rates: signal regenerators for digital transmission can more easily restore the signal to its exact original value, because the only possible values are 0 and 1 . High, single-channel data rates are possible, and the cost of digital transmission and associated switching continues to drop.

Disadvantages of digital signaling consist mainly of distance limitations associated with direst-turent baseband data transmissions. Over long distances, digital signals over a baseband rise and decay slowly due to impedance (capacitance and inductance) effects. For long-distance data transmission over coaxial and twisted pair (i.e., telephone wire), alternating current (ac)-based analog signaling is used.

Analog transmission uses an ac sine wave as the basis for representing data. Bit values are represented by one of three ways: 1) amplitude modulation, 2) frequency modulation, or 3) phase modulation. These three techniques are illustrated by Figure 2.4 .

Amplitude modulation uses different voltage levels to represent $1 \mathrm{~s}$ and $0 \mathrm{~s}$. Frequency modulation maintains the same amplitude but varies the frequency for 1 anc 0 bits. As the name implies, phase modulation changes t'ie phase of the sine wave to represent bit values

Advantages of : nalog signaling include the ability to transmit multiple channels over coaxial cable for distances of tens of kilometers, with amplifiers required about every 500 feet.

Disadvantages of analog signaling include greater difficulty in filtering out line noise and the need to

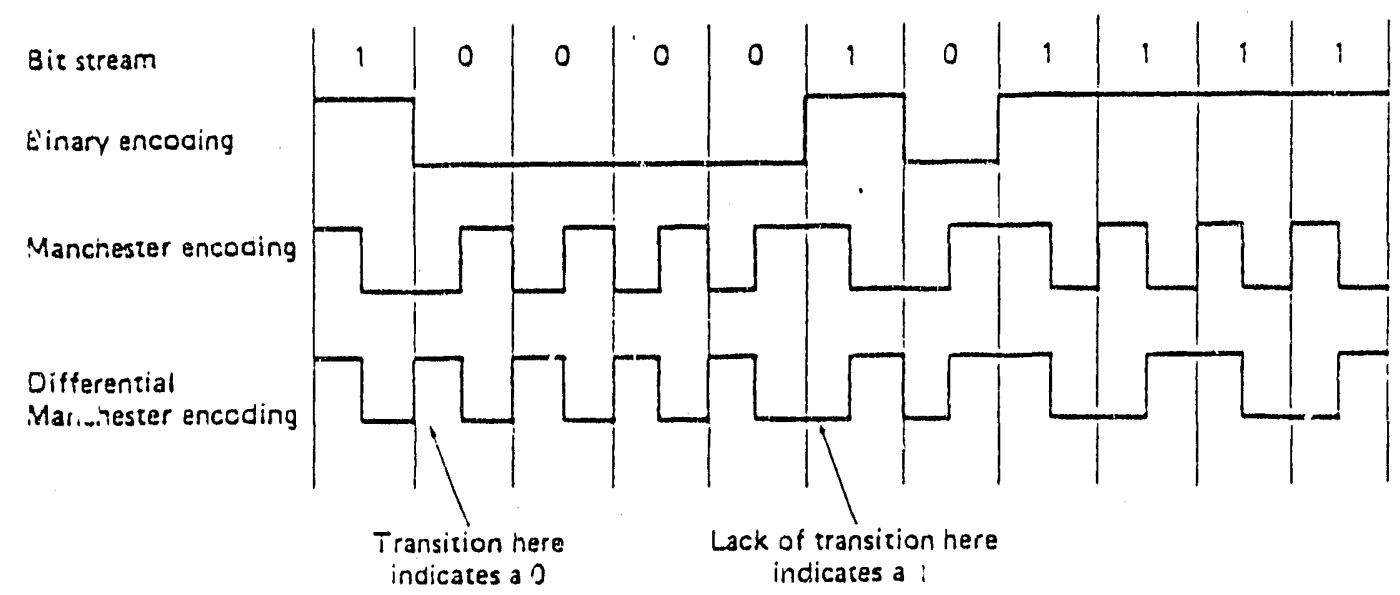

Figure 2.3. Digital Signal Encoding Techniques 


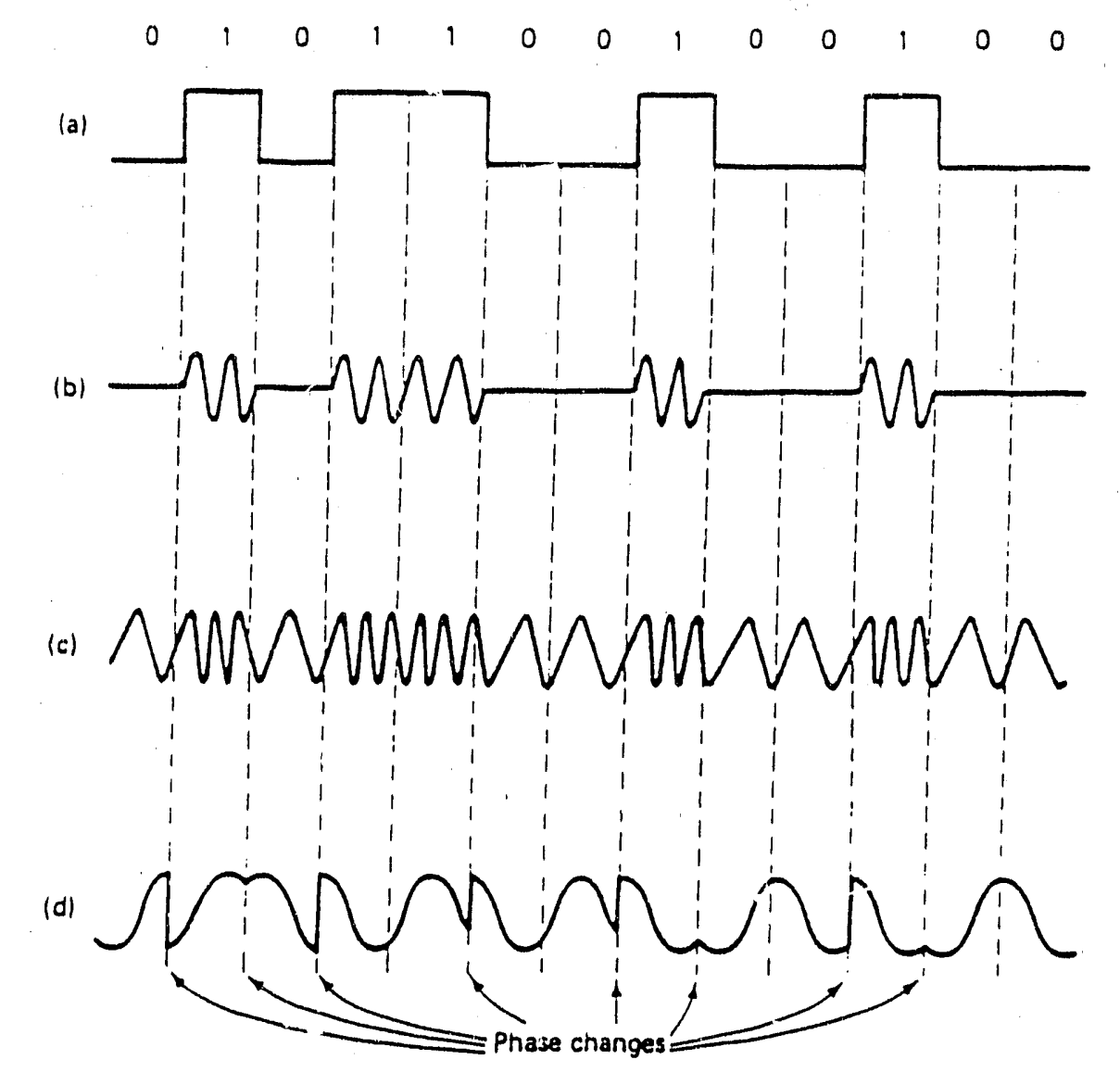

Figure 2.4. Analog Signal Encoding Techniques: a) Original Signal in Direct Current Form, b) Amplitude Modulation, c) Frequency Modulation, d) Phase Modulation

modulate digital bi: streams and demodulate the resulting analog signal at the destination (e.g., use of a modem or modulator-demodulator for sending computer files over phone lines). Another disadvantage of typical broadband based analog systems, as compared to digital systems, is the need for higher skilled personnel in the area of radio frequency engineering to plan and maintain the system. Finally, amplifiers for broadband-based ana!jg systems require po'ver, which is often difficult to provide for long stretcines between buildings:

\subsubsection{Transmission Media}

Transmission media refers to the variety of physical layer methods used for transporting bit information. Two common terms are baud and bandwidth. A baud refers to the number of times per second that a digital signal changes its value (e.g., voltage level). The baud value does not necessariiy equal the bits-persecond transmission rate. For example, if a binary signal were sent with 1 volt for a 1 bit and 0 volts for a 0 bit, every signal change would represent a new bit sent (baud rate $=$ bit rate). However, if multiple voltages were used to represent bits, each signal could convey multiple bits, resulting in the bit rate being some muitiple of the baud rate.

Other terms related to data transmission over physical media include simplex, half-duplex, and fullduplex transmission. Simplex refers to a media that Caus iscismit in one direction only. Hali duplex means the media can trad . mit in both directions but not at the same time. Full-dup'ex means that simultaneous transmission of data in both directions is supported $b$ : the media.

Characteristics of common transmission media are summarized in the following sections:

\section{Magnetic Media}

In short, magnetic media transmission refers to writing data onto floppy disks or tapes arid physically transporting the media to another computer for 
uploading. In the case of floppy disk transfer, this is often referred to as "Sneaker Net." Typical industry standard magnetic tapes can hold 180 megabytes ( 8 bits $=1$ byte). Because of the high volume of data storage potential of magnetic tapes, simply transferring tapes is the most efficient and cost-effective data transmission method in many instances where timeliness is not an issue.

Advantages of magnetic media data transmission are that it is cheap and reliable. Also, magnetic media data transmission has the potential for relatively high data security.

Disadvantages of magnetic media data transmission consist primarily of the time and labor required to physically download, transport, and upload the data.

\section{Twisted Pair Wiring}

Primarily used for phone lines, twisted pair wiring is the most common transmission media used today. It consists of two insulated copper wires about $1 \mathrm{~mm}$ thick, twisted to avoid the mutual electrical interference that two parallel wires can produce. Twisted pair wiring can be used for both analog and digital data transmission. Data transmission rates of 1 to $2 \mathrm{mbps}$ can be achieved for transmission distances of 1 to 2 miles, in many cases. However, a bit rate of 9600 bits/sec achieved by sending 4-bit groups at 2400 baud is more common.

Advantages of twisted pair wiring are that it can transmit signals for several miles without the need for amplification, and it is cheap and may already be installed.

Disadvantages of twisted pair wiring consist primarily of sensitivity to electromagnetic interference, resulting in high data-transmission error rates as compared to other media and relatively low data transmission throughput capacity.

\section{Baseband Coaxial Cable}

Baseband coaxial cable consists of a stiff copper core surrounded by an insulating material. Baseband is a 50-ohm cable used for digital transmission. As the name "base" implies, baseband only transmits one channel for the data transmission it supports. The Manchester rigital encoding techniques (Figure 2.3) are commonly used by this signaling method. The cable resembles yellow garden hose, with markings every 2.5 meters to indicate where taps should go. A typical application has a data transmission capacity of about
10 mbps. Baseband cable taps include both $\mathrm{T}$ junctions for thin ethernet cabling and vampire taps for thick ethernet cabling. A $T$ junction (referred to as a BNC) tap involves cutting the cable and inserting a tap that reconnects the cable, with a third wire leading off to a computer. A vampire tap involves drilling into the cable and screwing in a special connector, forming a $\mathrm{T}$ in the cable.

Benefits of baseband cabling are that it is inexpensive and easy to install, as compared to broadband cabling. Computer interface cards are less expensive than for a alalog-based systems, and the digital signals can pericatically be regenerated to ensure error-free transmission.

A disadvantage of baseband cabling is that the single channel prevents simultaneous transmission of voice, video, and data bit streams.

\section{Broadband Coaxial Cable}

The term "broadband" generally refers to any cable network using analog transmission (typically 75-ohm cable). Based on standard cable television technology, broadbands have a bandwidth up to 300 to $450 \mathrm{MHz}$ and can transmit data tens of miles. Each computer interface connected to the analog network must contain an interface or be connected to a shared interface that converts the outgoing bit stream to an analog signal and the incoming channel aradog signal to a bit stream. A $300 \mathrm{MHz}$ cable will support a total data rate in the range of $150 \mathrm{Mbps}$.

Broadband coaxial cable has three basic components: the solid core center conductor, the dielectric, and the outer conductor or shield. The center conductor usually consists of copper-clad aluminum, which nrovides the same transmitting characteristics of solia copper but at lower cost. The dielectric or insulation is usually made of a foamed polyethylene or polystrynene. The outer conductor or signal shield is usually a solid, extruded aluminum tube.

Broadband systems are typically divided into multiple channels, each of a specific frequency range. A primary difference between baseband and broadband systems is that broadband systems use amplifiers to strengthen the signal periodically. These cinplifiers can only transmit signals in one direction. Broadband systems, therefore, either require dual cables (one for incoming and one for outgoin(; signals) or a system design that divides a single cable into frequency bands for inbound and outbound con munications. With the single-cable concept, low frequency bands are used for 
outgoing signais from the computer through a low-frequency amplifier, to the network headend.

At the headend, this subsplit or midsplit system transforms low-frequency signal channels into resulting signals on high-frequency channels. These highfrequency channels transmit signals from the headend through any high frequency amplifiers that might be required and on to the destination computer. Between each frequency channel is a range of buffer frequencies not used (i.e., guard bands), which minimizes interference between channels. This concept is also referred to as frequency-division multiplexing, and each terminal is assigned an inbound and outbound channel.

For improved reliability, broadband headends should include a fully redundant system and emergency power backup.

Analog signaling on coaxial cable when only one channel is used is called "carrier band." Carrier band cabling is used to support MAP and other token bus topologies on the factory floor.

Benefits of broadband cabling include the ability to transmit high-volume data streams over long distances. Also, broadband as a network backbone enahles an installation to transmit data, voice, and video signals over the same network cable.

Disadvantages of broadband cabling include higher computer interface costs, as compared to baseband, to accommodate analog signaling. Also, the system requires more sophisticated support, and accumulative signal errors may occur at longer distances.

\section{Fiber Optics}

In simple terms, fiber optics uses a light pulse to signal a 1 bit and the absence of a pulse for a 0 bit. The frequency of visible light is about $10^{8} \mathrm{MHz}$, providing a potentially enormous data communication bandwidst.

A fiber optic system consists of three basic components: the transmission medium, the light source, and the detector.

The transmission medium is a thin fiber of glass or fused silica. The principle allowing these fibers to transmit light and not let it simply 'leak' out is based on refraction. When light passes from one medium to another (e.g., tused silica to air), the ray is bent or reflected. For certain conifigurations of media and light sources, all of the light is reflected back into the silica and none of it escapes into the air. In this manner, light can propagate for miles within a fiber at virtually no loss. A multimode fiber refers to different rays $\mathrm{br}$ uncing at different angles through the fiber. Reducing the fiber's diameter to one wavelength of light results in the fiber's acting as a wave guide, meaning the light propagates in a sisaight line (referred to as a "single mode fiber"). Single-mode fibers must be driven by expensive laser diodes, but they are more efficient and can transmit light for longer distances than multimode fibers.

Multimode fibers are driverı by light-emitting diodes (LEDs) or by lasers, which emit light pulses when electric current is applied. Light pulses are converted back into electric pulses by the use of a photodiode. Commercially available fiber optic systems can transmit data at about $1000 \mathrm{Mbps}$ (at one mile).

Taps for fiber optic systems are more complex and expensive than for coaxial cable systems. Vampire taps that fuse a fiber lead to form a $\mathrm{T}$ result in substantial light loss. A more common tap is the active repeater tap, shown by Figure 2.5, used in ring configurations. Incoming light signals are translated into an electrical signal via a photodiode, and the signal is regenerated to its original strength. A copper wire lead from the connecting terminal is attached at this point. Following the electronic transmission of the signal to and from the connecting terminal, the signal is then converted back to light pulses via a light-emitting diode or laser, and the transmission continues.

As the signal is regenerated to its original intensity at each tap, there is virtually no limit on the total size of the ring. Failure of any one tap will bring the entire ring down; however, redundancy can be built in to reduce this drawback.

Fiber optic networks can also be built without the ring configuration, as shown by Figure 2.6. A device known as a passive star broadcasts each light signal to every terminal and is fed by every terminal. Each terminal checks the broadcasted signal to determine if it is addressed for that terminal. All of the incoming signals are divided among all of the outgoing lines, meaning that the sensitivity of the photodiodes determine the maximum number of allowable nodes in the network. 


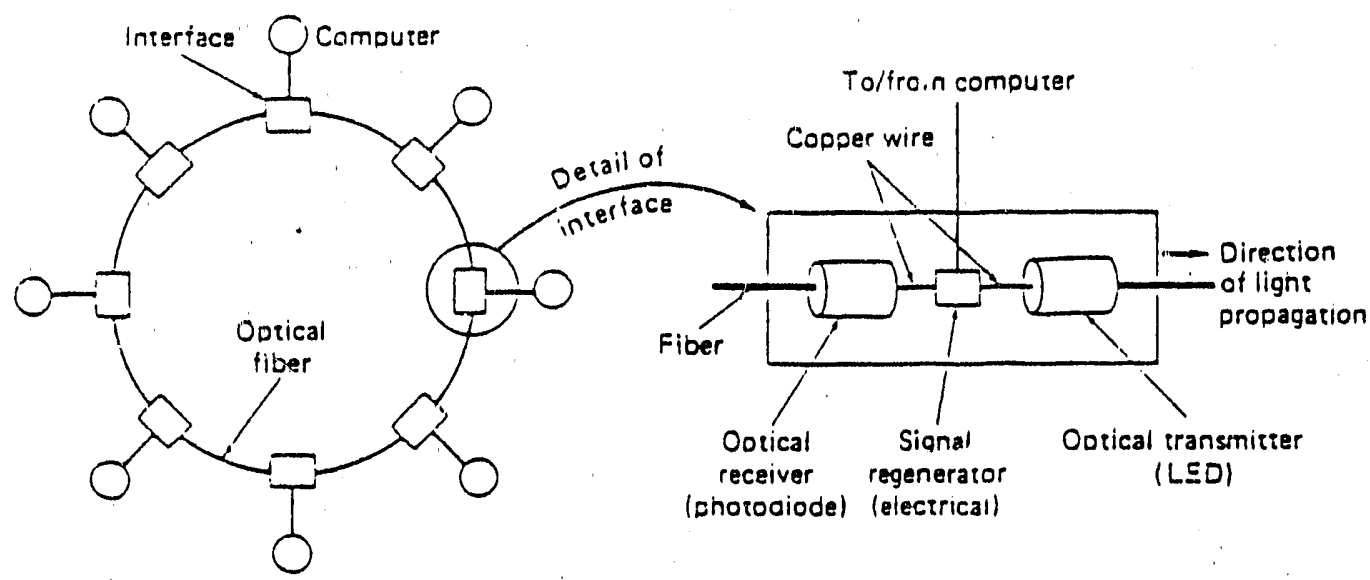

Figure 2.5. Fiber Optic Active Repeater Tap

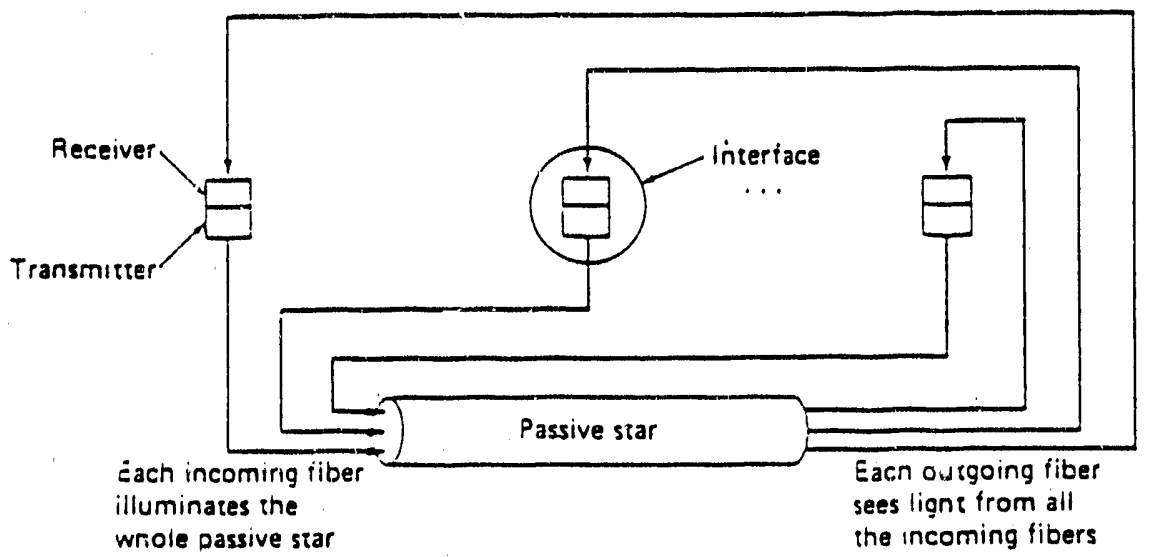

Figure 2.6. Fiber Optic Passive Star Configuration

Benefits of fiber optics, as compared to coaxial cable, include the ability to support higher data transmission rates at longer distances. Fiber optics are not affected by power line surges or electromagnetic interference. Fibers are also thin and require minimal room in cable ducts.

Disadvantages of fiber optics, as compared to coaxial cable, are that it requires technology unfamiliar to most on-site engineers, and interfaces are expensive.

\section{Microwave Transmission}

Microwave transmission is a line-of-sight communication system based on microwave radio transmission. Most microwave transmissions occur at frequencies between 2 and $40 \mathrm{GHz}$, corresponding to wavelengths of 15 and $0.75 \mathrm{~cm}$. Parabolic antennas are mounted on buildings or towers to send a beam to a receiving tower, which can be tens of miles away. Related techniques include the use of infrared and laser signals.

Benefits of microwave signaling, as compared to coaxial cable, are that it may be cheaper and easier than pole-mounted or buried cabling, and have less potential for damage to the transmission equipnent.

The primary disadvantage of microwave transmission is the line-of-site requirement between the transmission and receiving towers.

The primary disadvantage of microwa e signaling is that signals from a single antenna may split up and propagate by slightly different paths to the receiving antenna, reducing signal strength. Thunderstorms and other atmospheric phenomena also can interfere with microwave signaling. 


\subsubsection{Network Components}

A variety of network components exist for extending the distance capability of local area networks (LANs) and/or integrating dissimilar networks. The use of these components as part of the migration strategy to GOSIP/OSI compliance is presented in Chapter 4.0. Descriptions of these essential network components are given in the following paragraphs.

\section{Repeaters}

A repeater forwards bits from one network to another, making the networks look logically like one network. A repeater, as shown by Figure 2.7, operates only at the physical layer of the OSI model (i.e., layer 1). Repeaters are simple devices and contain minimal software. A typical use of a repeater is to extend the length of an Ethernet-based LAN. Unbuffered repeaters on digital networks are similar to amplifiers on analog-based networks, in that the incoming signal plus any signal noise is strengthened or amplified and forwarded along the network. Buffered repeaters differ from unbuffered repeaters in that the incoming bit signal is interpreted and a new clean signal is generated, thus eliminating any signal noise.

\section{Bridges}

A bridge operates up through the data link layer of the OSI model, as shown by Figure 2.7. A bridge can be used to connect two networks that have different data link layers but the same network layer. For example, a bridge used to connect an Ethernet LAN to a token ring LAN would receive data frames in Ethemet form and copy the frames into token ring form, or vice versa.

\section{Routers}

A router operates up through the network layer of the OSI model, as shown by Figure ' 7 . A router is used for connecting two networks that 1 ave the same transport layer but different network layers. For example, a connection between a token bus and an X.25 public network (i.e., phone lines) would need a router to convert the token bus frames to the form required by the X.25 network. A router, similar to a bridge, filters incoming signal traffic and only passes those signals destined for the connecting network.

\section{Gateways}

A gateway operates up through the transport layer + and higher, depending on the application. Gateways serve to integrate networks that can be entirely different, from the physical layer up through the application layer, as shown by Figure 2.7. For example, an IBM SNA network can be connected to a TCP/IP network using the appropriate gateway. A gateway can be thought of as a computational server similar to a 386 PC, with two interface cards (i.e., one card for each type of network served) and protocol translation software. Like routers, gateways can filter network traffic.

\subsubsection{Related Information System Ter- minology}

Modernization planners wading through network terminology will also be confronted with a multitude of other terms related to information system modernization. This section briefly describes some of the most common terms related to plant-wide integrated information systems.

\section{OSI Virtual Terminal Protocol}

In any large network, multiple types of terminals exist that will need to communicate with each other. The OSI Virtual Terminal (VT) protocol assists in integrating heterogeneous terminals within a network. A typical VT application is where a remote terminal user wishes to access some application program, such as a full-screen editor. The application is typically expecting to interact with only a lim ited set of terminal types. However, if the user's terminal is not among those directly supported by the application program, some kind of mapping is required between the control functions assumed by the application program and those actually supported by the user's terminal.

The OSI Virtual Terminal protocol completes this mapping by defining a "virtual terminal," an abstract representation of a real terminal. (See Figure 2.8).

The remote minal side of the connection maps its own terminal type to the virtual terminal, and these virtual terminal protocols are then mapped to a terminal type supported by the application. Rather than defining a single virtual terminal for all possible 
Layer

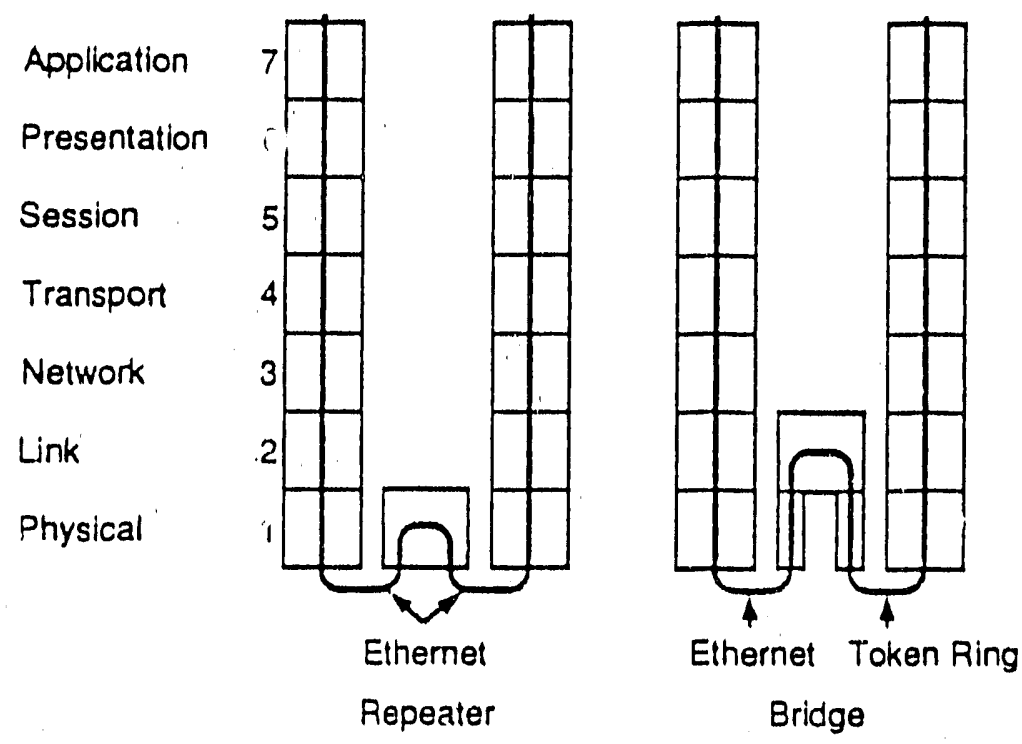

Layer

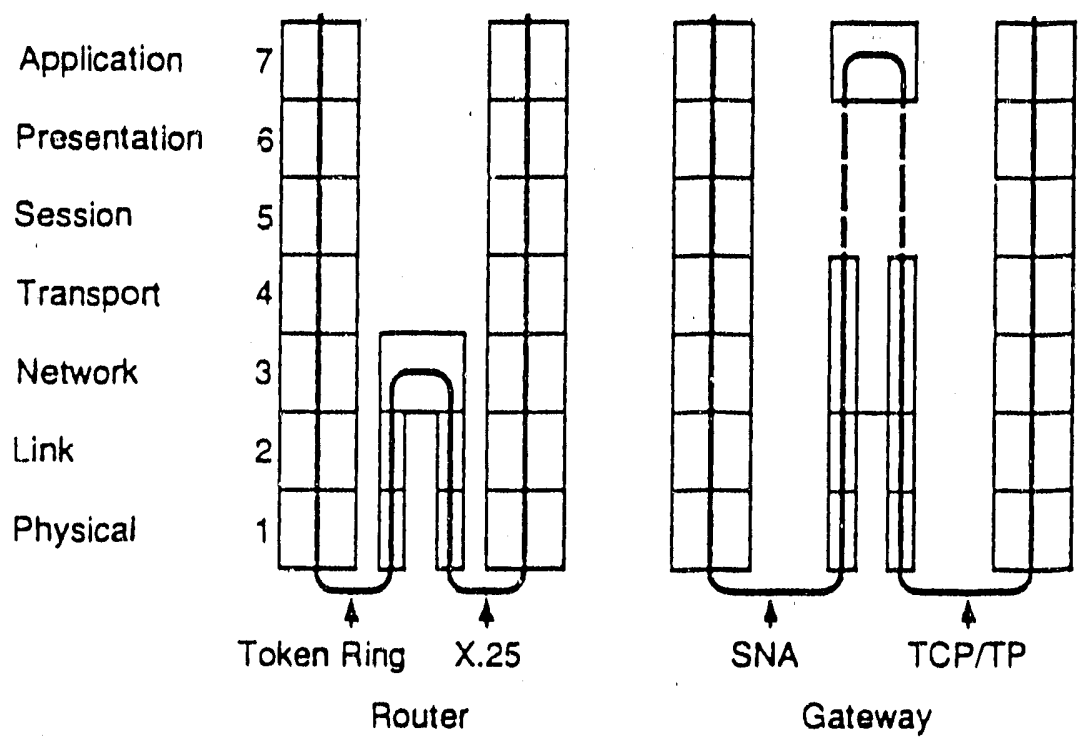

Figure 2.7. Network Components and the OSI Model

connections, the virtual terminal protocols provide the tools to define an unlimited number of virtual terminals, depending on the need.

\section{X.400 Message Handling Standard}

The 1984 CCITT X.400 message handling standard has be in accepted by OSI as the standard governing OSI layer-7 electronic mail systems. The intent of this standard is tc ensure compatibility between all message handling system software that adopt the
X.400 protocols, thus eliminating the need for translation software to integrate incompatible message handling systems.

\section{X.500 Directosy Services}

The 1988 CCITT X.500 directory system standard is an OSI layer-7 system for maintaining up-to-date information about all people, prograrns, and various shared network resources using the communication 


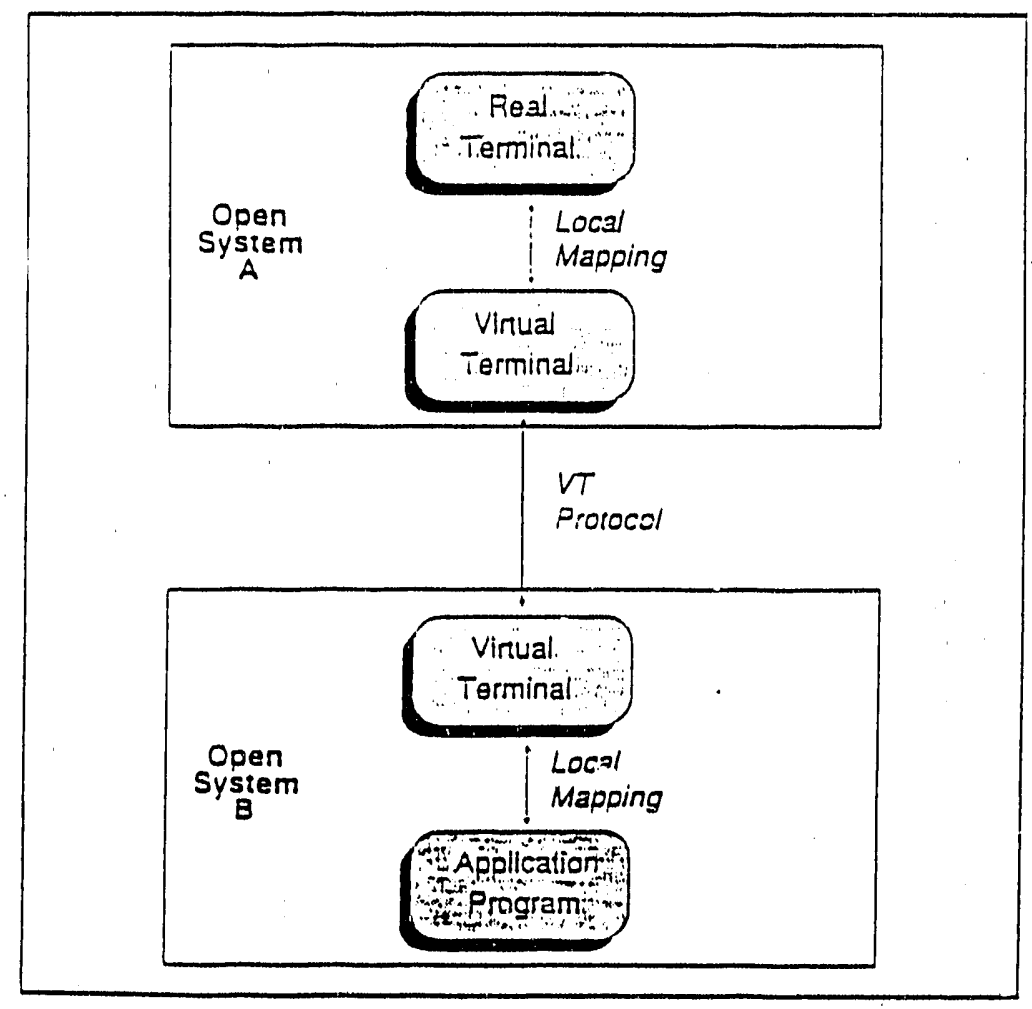

Figure 2.8. Virtual Terminal Concept

system. This standard supports both network managers and message handling systems.

\section{File Transfer, Access and Management (FTAM)}

File Transfer, Access and Management (FTAM) is the OSI layer-7 standard for file transfer, in existence since 1987. This standard describes a service and its respective protocols that allow users to read or write files in a remote file store. The need for FTAM stems from the broad range of file types and styles used by data management systems. To effectively manage these diverse file types within the OSI environment, FTAM was designed to translate or map file types into a general file model or virtual file store. Using a concept similar to that used by virtual terminals, files are mapped to FTAM's virtual file store using a hierarchical file structure known as a File Access Data Unit (FADU). FTAM-compatible products are being pursued by multiple vendors and system houses, and their usage is expected to increase dramatically. release.

\section{Product Data Exchange Specification (PDES), IGES, EDI}

The Product Data Exchange Specification (PDES), currently being developed under the supervision of the National Institute of Standards and Technology (formally National Bureau of Standards), has the objective of defining what constitutes a complete product definiun $n$. The fundamental idea of PDES is that there is a common set of data that describes all products, independent of specifis graphical packages or computer environments. Once this standard product data definition (referred to as the integrated product data model) is established, it can be used to "map" specific computeraided design packages to and from the standard model. This concept, once again similar in nature to the virtual terminal concept, will provide the enabling technology for integrating electronically diverse engineering work stations and their respective drawings, product specifications, maintenance requirements, and related test systems. PDES is, in a sense, an expansion of the CAD standard for the Initial Graphics Exchange Standard (IGES), an information exchange protocol for engineering design drawing secking to standardize the preparation of engineering data. 
The business world equivalent of IGES is the Electronic Data Interchange (EDI) standard, a set of standard formats describing the information content of business documents such as purchase orders, funds transactions, etc. A promising area being investigated is the integration of EDI- and X.400-compatible products, resulting in electronic business 1 ,rms that could be transmitted and read electronically. 


\subsection{NETWORK TOPOLOGIES}

\subsection{OVERVIEW OF NETWORK CONFIGURATIONS}

Network topologies or architectures refer to the configurations used to connect nodes or stations within a communication network. Network topologies, as shown by Figure 3.1, may be grouped into the following general classes: bus, ring, star, and mixed.

A common architecture for LANs is the bus topology, which consists of communication stations attached to a common backbone link.

The ring topology is another common LAN architecture. Communication stations are linked to form a ring or loop.

Star topology is often referred to as an "cstopus" architecture, where links from communication stations are all connected to a centralized unit. This configuration is typified by mainframe systems fed by dumb terminals via twisted pair wiring.

Finally, mixed topologies represent any combination of bus, ring, or star configurations. This is a common feature in most large organizations, especially where large systems have been created through piecemeal planning and funding. Exanples of LANs based on these general classes of netwurk topologies are presented in the following sections.

The three LAN standards currently recognized by GOSIP in FIPS 146 are:

- IEEE 802.3 CSMA/CD (Ethernet)

- IEEE 802.4 Token Bus

- IEEE 802.5 Token Ring.
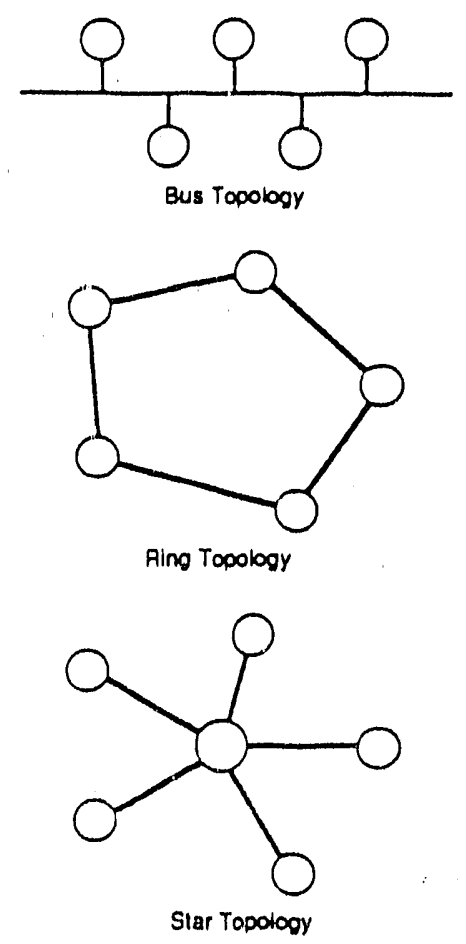

Figure 3.1. Network Topologies 
These IEEE standards specify the physical layer and the lower sublayer of the data link layer within the OSI reference model.

\subsection{IEEE 802.3 CSMA/CD (ETHERNET)}

Carrier sense multiple access with collision detection (CSMA/CD) is a LAN standard describing a bus type topology typically using 50-ohm baseband coaxial cabling rated at $10 \mathrm{Mbps}$. The IEEE 802.3

CSMA/CD standard specifies a family of CSMA/CD standards, with the term "Ethemet" referring to a specific product compliant, with IEEE 802.3. Thus, the terms Ethernet and IEEE 802.3 are not necessarily the same, but are often used interchangeably. This section will discuss the Ethemet implementation of the IEEE 802.3 standard.

The term "Ethernet" originates from an early CSMA/CD system developed by Xerox in the 1970s. and was derived from luminiferous ether, a substance physicists in the 1880s once thought was required to transmit electromagnetic radiation. The successful 1970s Ethernet work led to the development of a 10-Mbps Ethernet standard drawn up by Xerox, DEC, and Intel, which formed the basis of the IEEE 802.3 standard.

Very simply, when a node or station wants to transmit, it listens to the cable (i.e., carrier sense multiple access feature). If the cable is idle, the station transmits immediately. Should two stations simultaneously transmit, a collision will be detected (i.e., collision detection feature). All colliding stations terminate their transmission, wait a random length of time, and repeat the process.

Ethernet uses straight Manchester digital encoding, as does all the IEEE 802.3 implementations. The typical configuration of an Ethernet LAN connection is shown by Figure 3.2. The transceiver is clamped onlu the LAN network cable, making a tap with the inner core. The transceiver contains the electronics that handle the carrier detection and collision detection. Some transceivers can support up to eight resources, reducing the number of transceivers needed for any given network. The transceiver cable connects the transceiver to the interface board in the computer, which contains a controller chip that transmits frames to, and receives frames from, the transiciver.

The IEEE 802.3 standard under which Ethemet falls specifies a maximum LAN cable length of 500 meters. Longer cable lengths can be obtained by connecting multiple cables with repeaters. The entire cable length can be extended to up to $2.5 \mathrm{Km}$ between transceivers and a maximum of four repeaters. Beyond this limit, the CSMA/CD is no longer reliable unless bridges, routers, and/or gateways are used.

The performance of Ethernet-based LANs is not deterministic, as the performance of the LAN is determined by the amount of communication traffic at any one time. As traffic increases, more collisions are detected, resulting in more signals being present, which results in yet more traffic. Therefore, it is impossible

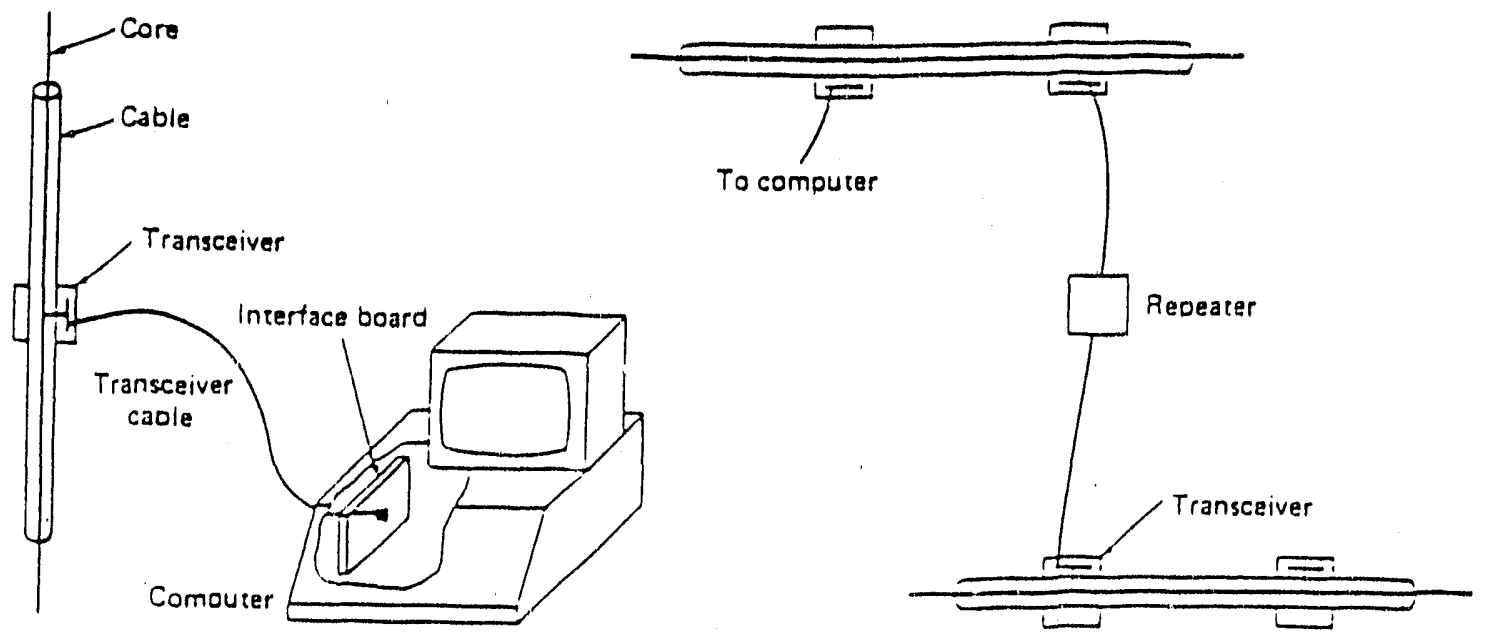

Figure 3.2. Ethernet LAN Connection 
to achizve $100 \%$ efficiency on a $10-\mathrm{Mbps}$ rated system. A rule of thumb is that a good, effective capacity of an Ethernet system is about 2 Mbps. For Ethernet I.ANs operating within this constraint, response time is superior to that of most other LAN configurations.

\subsection{IEEE 802.4 TOKEN BUS}

Although the IEFE 802.3-based LAN is the most widely used LAN in office environments, General Motors and other companies pursuing LAN technologies in support of factory automation had serious reservations about its performance. Specifically, response times on an IFEE 802.3-based system do not have a known upper bound, and stations cannot be assigned a priority for gaining network access. Both of these features are important in the design of real-time equipment control systems. However, the bus confizuration of IEEE 802.3 was preferred by General Motors and others over a ring configuration, because most assembly lines have a linear topology. The result of these considerations was the development of the IEEE 802.4 token bus standard in support of General Motors' Manufacturing .Automation Protocol (MAP) network communication standar1.

The IEEE 802.4 token bus standards are based on analog signaling using 75-ohm broadband coaxial cable. Standards support communication speeds of 1,2 , and $10^{\circ} \mathrm{Mbps}$. The general configuration of a token bus LAN is shown by Figure 3.3.

As shown by Figure 3.3, stations are attached to a linear broadband cable. Each station is assigned an address, and knows the address of the station "upstream" and "downstream." This results in stations being organized into a logical ring. The station with the highest priority may send the first data frame. When it is done or has no data to send, it passes permission to the next priority station by sending a special control frame called a token. The token moves around the logical ring, and only stations with the token can transmit. Because only one station at a time can transmit, no data collisions can occur. The maximum amount of time any particular station will have to wait for a token can be calculated. It should be noted that all data frames are broadcast to all stations, and stations discard data frames not addressed to them. Each time a station acquires the token, it can transmit data frames for a certain period of time. Priorities and times can be set to ensure that critical stations receive access to the network as needed.

Whereas the utilization of token bus LANs can achieve nearly $100 \%$, these LANs have more overhead in the data frames and somewhat slower response times than Ethernet LANs utilized at a typical value of $40 \%$.

\subsection{IEEE 802.5 TOKEN RING}

Ring networks have been around since the early 1970s, and essentially consist of a collection of individual point-to-point links that happen to form a circle. Multiple types of ring networks exist, ranging from fiber optic to twisted pair-based rings. The token ring network specified by IEEE 802.5 is compatible with IBM's LAN architecture. This particular token ring network uses digital signaling on shielded twisted pair wiring running at 1,4 , or $16 \mathrm{Mbps}$. Signals are encoded using differential Manchester encoding. A token ring network is illustrated by Figure 3.4.

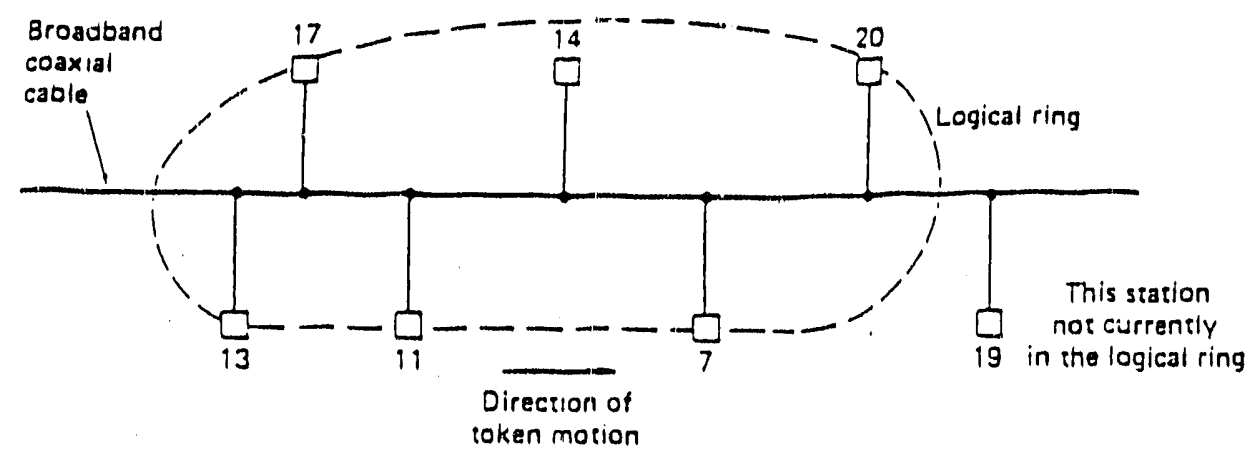

Figure 3.3. Token Bus Configuration 


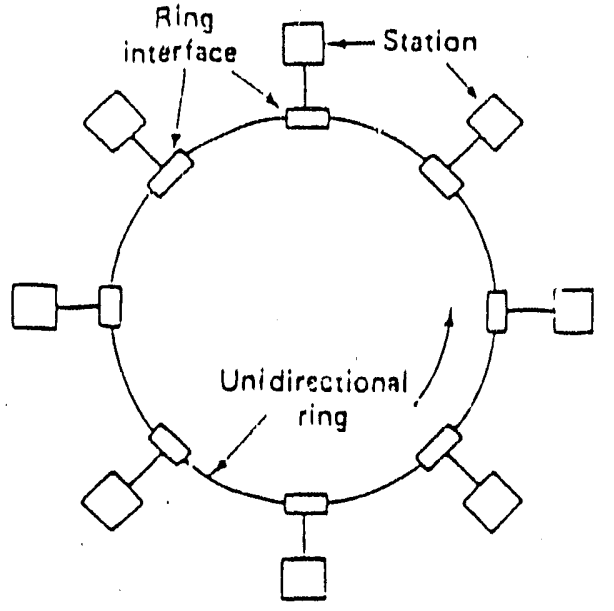

Figure 3.4. Token Ring Configuration

Stations attempting to use the network are granted access when a data element called a token becomes available. A station wishing to send information grabs the token and modifies a bit in the token data element, changing the token bit pattern to a start-offrame bit pattern. The sending station then adds its own data elements (1) the start-of-frame pattern and sends the data on the wire. The frame is sent from station to station. Each station inspects the address and forwards the message to the next station in the path if it is not the intended receiver of the message. When the message reaches the targeted station, it is copied to the station and forwarded back along the network to the sender. The sender can then check to see if the data frame was received correctly. When the last bit of the frame has gone around and come back, it is removed and the token data element is regenerated and forwarded to the next station. There is no required limit on the size of a data frame that can be transmitted, because the entire frame never appears all at once on the ring.

Communications from the ring to the individual stations are handled by the ring interfaces, as shown by Figure 3.4. Ring interfaces have two modes, "listen" and "transmit". I the listen mode, incoming bits are simply copied to uutput. In the transmit mode, the data is received if it is intended for the station, and the token and data frame is copied back to sender; or if the station wishes to transmit, the token is seized and the station's own data is entered on the ring.

One of the problems with ring networks is that a single cable break will cause the whole ring to malfunction. Most 802.5 LANs use a concept called a wire center or star-shaped ring to improve ring reliability, as shown by Figure 3.5 .
Each station is connected to the wire center by a minimum of two twisted pair wires or fiber cables, one for data to the station and one for data from the station. Bypass relays within the wire center are powered by current from the stations. If the ring out to the station breaks, the relay will bypass the station and protect the ring. Ring reliability can also be improved by the use of counter-rotating rings, as described in Section 3.8 .

\subsection{COMPARISON OF 802 LAN STANDARDS}

Each of the three 802 LAN standards has strengths and weaknesses, depending on the application. IEEE 802.3 CSMA/CD is presently the most widely used LAN. Stations can be installed without taking the network down, and modems are not required. At low utilization, the delay for gaining network access is practically zero, because stations do not have to wait for a token. Advantages of IEEE 802.4-token bus LANs are that they are deterministic and support the assignment of access priorities to stations. Token bus LANs also show excellent efficiencies at high utilization rates. Shortcomings of 802.4 based LANs, however, are that they require significant analog ingineering, including modems, and have a certain amount of delay at low load. Finilly, 802.5 LANs are deterministic, cheap, and easy $t u$ install. However, interface cards for 802.5 LANs are more expensive than for 802.3 LANs.

Competing with the IEEE 802 LAN series are a variety of other network options, as described in the following sections.

\subsection{SLOTTED RINGS}

A slotted ring is similar to a token ring configuration, with the exception that more than one station can be transmitting at once. This is accomplished by having the signals on the ring slotted ur divided into a number of fixed size frames, as shown by Figure 3.6.

Each frame, somewhat similar to a token, contains a bit that tells whether it is full or empty. A station wishing to transmit simply waits for an empty frame to come around, marks it as full, and puts its data into the frame. Unlike token rings, each frame has definite limitations on the amount of data that can be held. 


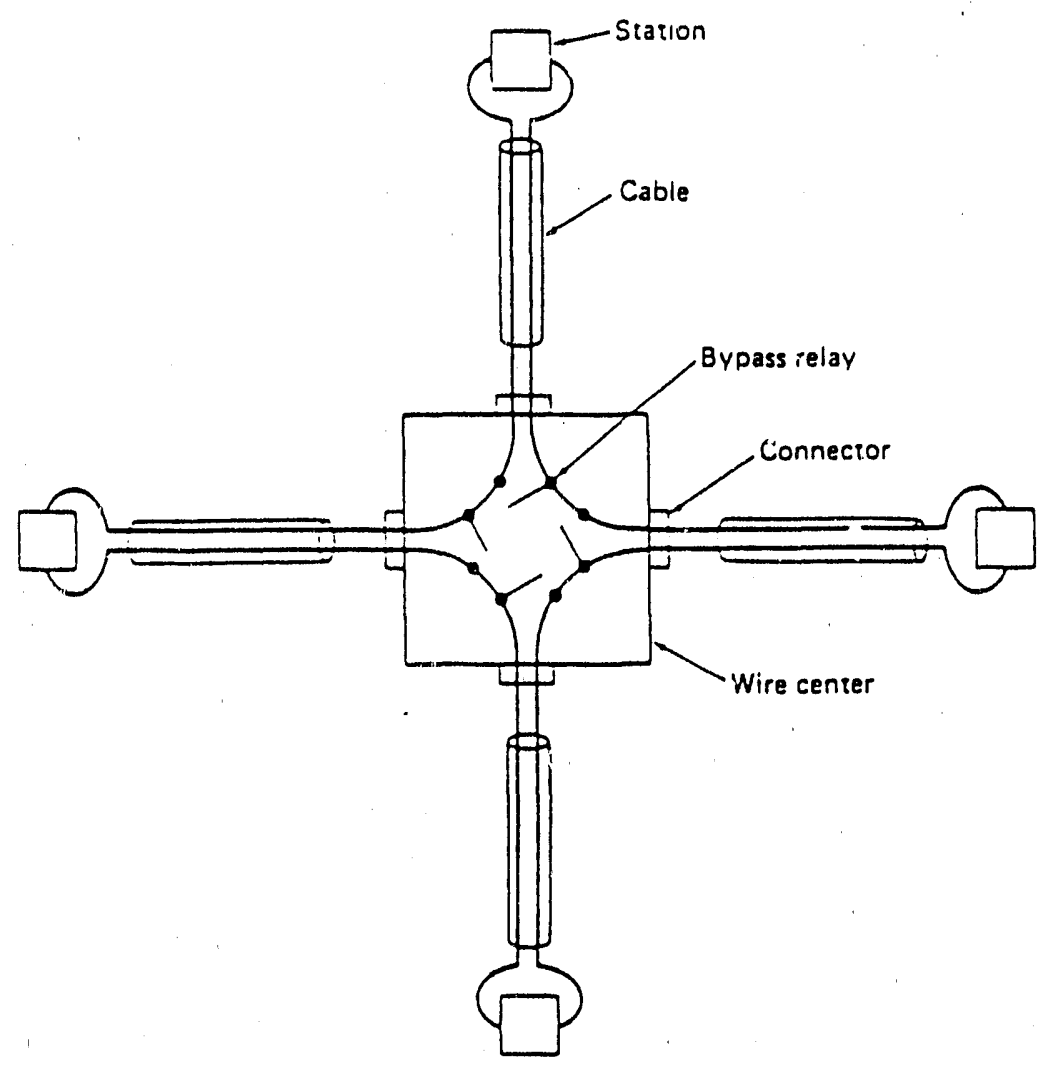

Figure 3.5. Wire Center for Improved Ring Reliability

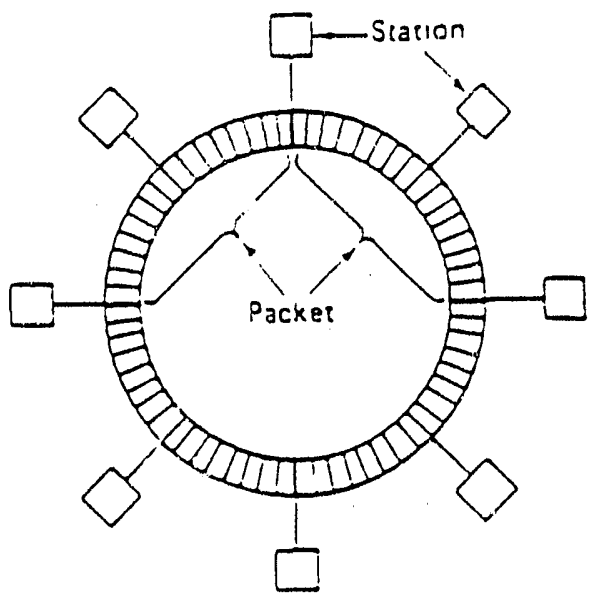

Figure 3.6. Slotted Ring Configuration

\subsection{PRIVATE BRANCH EXCHANGE (PB X)}

Telephone switching technology, in the form of private branch exchanges (PBXs), will compete with
LAN technology for the indefinite future. Very simply, PBXs are based on phone lines and switching circuitry to perform the functionality of LANs, as shown by Figure 3.7.

Advantages of using PBXs for networking stations are that existing telephone wiring can be used, and connecting the system to a wide area network is greatly simplified. The primary deficiency of a PBX network, however, is the $64 \mathrm{Kbps}$ bandwidth limitation of each channel, severely limiting the speed of any type of major data transmission. The result is that although a PBX may have a total rating of $500 \mathrm{Mbps}$, most channels are not used at any one time, and the channels that are used can not gain access to the excess capacity. This may result in a PBX-based system with a lower throughput than a 10 Mbps LAN.

An ongoing trend is to use PBX-based technology for wide area networking and LAN technology for inhouse traffic. This architecture relies on the use of gateways to integrate internal LANs with outside traffic. 


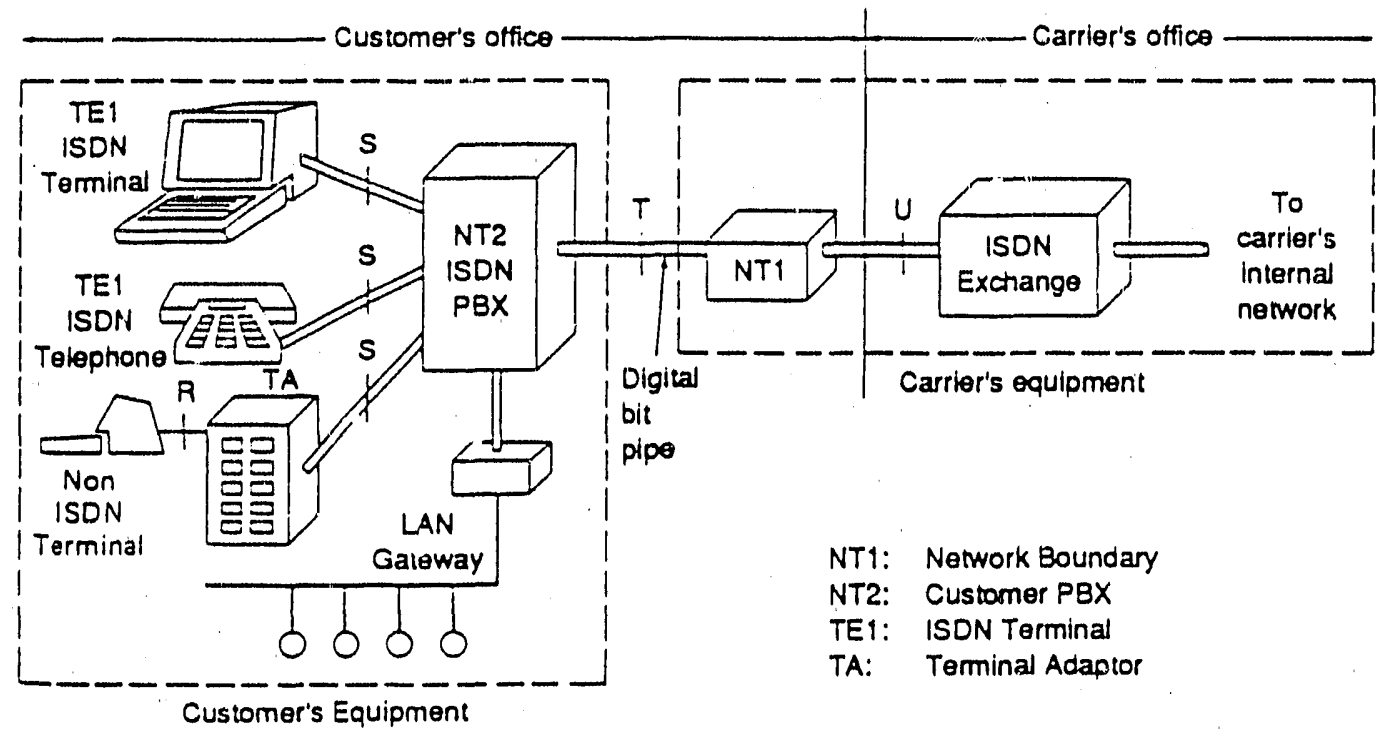

Figure 3.7. Communication System Using Private Branch Exchanges

\subsection{FIBER OPTIC NETWORKS}

A wide variety of fiber optic networks exists, duplicating many of the topologies using copper media that have previously been discussed. For example, the fiber-based network FIBERNET II is compatible with Ethernet at the transceiver interface. The fiber optic LAN known as Fiber Distributed Data Interface (FDDI) standards, adopted by the American National Standards Institute (ANSI), is scheduled to be recognized by the next GOSIP release.

The FDDI is a fiber optic, slotted ring LAN running at $100 \mathrm{Mbps}$. Individual fiber runs may be up to $2 \mathrm{~km}$ between stations, with a total ring length of 100 $\mathrm{km}$. Up to two rings can be connected for a total LAN distance of $200 \mathrm{~km}$, connecting up to 500 nodes or stations per ring.

Implementations of FDDI typically use multimode fibers and light-emitting diodes (LEDs). The cabling consists of two fiber rings, one transmitting clockwise and the other transmitting counter clockwise, as shown by Figure 3.8.

If either ring breaks, the other ring can be used as a backup. Or, if both rings break at the same point, (for example, because of some mishap in the cable duct), the two rings are automatically joined via repeater switching into a single ring approximately twice as long, as shown by Figure 3.8. Stations contain relays that can be used to join the two rings or bypass the station. Class A stations connect to both rings and have the highest reliability; Class B stations are cheaper and only connect to one of the rings.

FDDI protocols closely resemble the 802.5 token passing scheme. One primary difference, however, is that a 802.5 station generates a new token only when its frame has gone all the way around and back. With FDDI, stations generate a new token as soon as they are finished transmitting their frames; thus, several frames might be on the ring at any one time.

\subsection{PLANT-WIDE TOPOLOGIES}

Most industrial installations do not consist of one large building, but rather dozens to hundreds of buildings distributed over significant acreage. The mission and activity of each building will vary significantly, ranging from high-activity production and administrative buildings to low-activity warehouses and storage facilities. This results in each building having a LAN topology best suited for its particular activity level and mission. For example, production areas might best be served by token bus LANs if real-time control is involved. Administrative areas might dictate Ethernetbased LANs for office applications. Low-activity warehouses might be adequately served by token rings or the use of phone lines and modems to send data to the balance of the plant-wide network. 

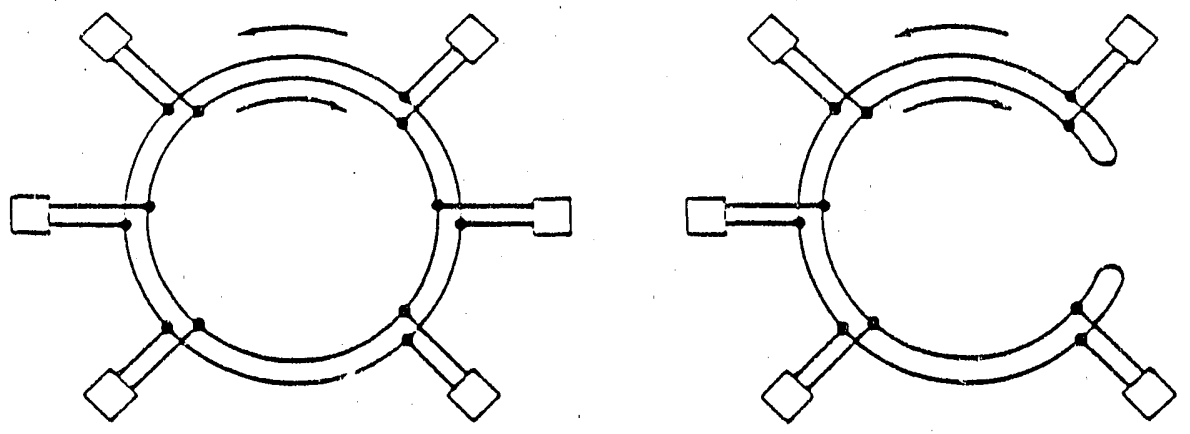

Figure 3.8. Ring Configuration Using Fiber Distributed Data Interface

With each building having a particular LAN best suited for its own function, multiple small LANs can be expected in any large industrial complex. To integrate these individual LANs into an effective plantwide communication network requires that the LANs be connected to some type of backbone network extending from building to building. For adequate sys. tem response, the interconnecting or backbone netwoik should be faster than the networks it interconnects or the direct systems connected to it.

Two candidates for providing the high-speed backbone functionality are fiber optics and broadband cabling technology. Bridges, routers, and gateways are used to integrate smaller, dissimilar networks onto the backbone to provide for one integrated plant-wide communication system. Broadband backbones are typically configured in a bus topology, with the single cable handling both incoming and outgoing signals via frequency division multiplexing. Fiber optics-based backbones, which are expected to increase significantly in use (Hancock 1989), use both ring and bus topologies for backbone design. An example of an FDDI ring being used as a backbone is shown by Figure 3.9.

The installation of a communication backbone represents an early infrastructure investment of any installation seeking plant-wide integration. Representative costs for communication backbones for large, diverse installations range from $\$ 500,000$ to $\$ 2,000,000$ and provide the foundation for integration.

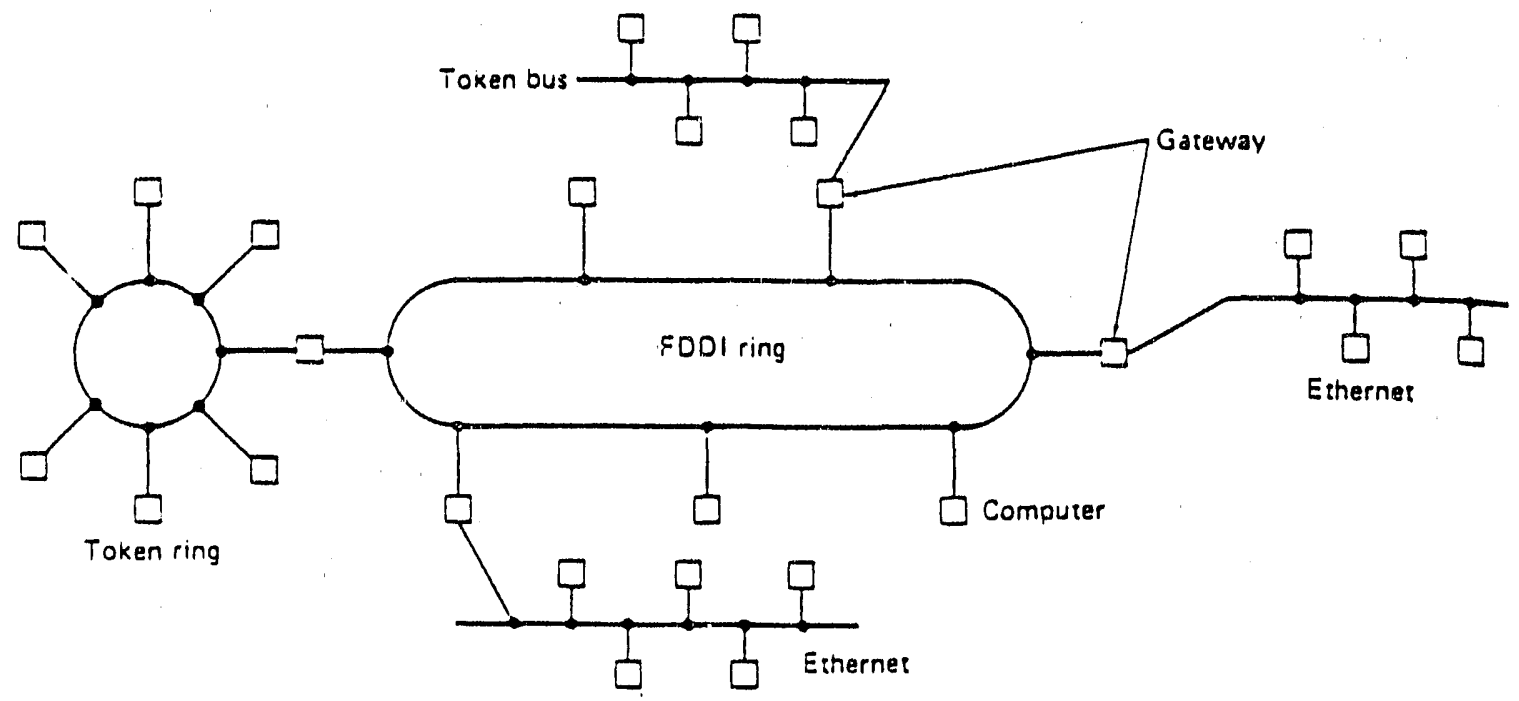

Figure 3.9. FDDI Ring Used as a Backbone to Connect LANs and Computers 


\subsection{NETWORK MIGRATION STRATEGIES}

\subsection{MEETING NEAR- AND LONG-TERM NEEDS}

In the mid-1990s, the Government Open Systems Interconnection Profile (GOSIP) is expected to he fully effective, requiring new hardware/software procu $\mathrm{n}$. ments to be fully OSI compliant. Until then, however, installations seeking to improve their manufacturing competitiveness and business practices need to pursue plant-wide integration using the best available commercial products. These commercial products [such as Transmission Control Protocol/Internet Protocol (TCP/IP), which provides the basic functionality of layers 3 through 6 of the OSI reference model] are not all GOSIP/OSI compliant. What is needed is a network migration strategy allowing installations to take advantage of the best commercially products currently available, while meeting GOSIP requirements as compliant products mature.

\subsection{PHASING IN OSI-COMPLIANT TECHNOLOGY}

A successful network migration strategy will allow all products to be used for their entire effective economic life. The essence of this migration strategy is a gradual substitution of non-OSI layers with OSIcompliant layers until only OSI products are left within the communication system. Simply replacing non-OSI-compliant hardware/software with OSIcompliant technology before the economic life of the non-OSI product is "used up" is not cost effective. Rather, the migration should be accomplished by essentially doubling up on the use of OSI and non-OSI products in some places and "unplugging" non-OSI products as it becomes economically feasible to do so. This is accomplished by putting in parts of both protocol stacks in key places and running both sets of protocol suites in parallel, after which non-OSI protocols are eventually dropped. Running OSI and non-OSI protocol suites in parallel is currently possible, using specially designed bridges, routers, and gateways that translate non-OSI protocols into OSI protocols, and vice versa.

In the case of TCP/IP, software exists that will allow OSI layer-7 application products, such as X.400 and FTAM, to run on top of existing TCP/IP net. works. This code, referred to as ISODE for ISO Development Environment, makes the OSI application behave as though it is running on top of an OSIcompliant network while making the TCP/IP network behave as though the application is TCP/IP-compatible. In this manner, OSI layer-7 applications can be run across the entire plant-wide network consisting of both TCP/IP and OSI protocols, further assisting the migration strategy to full OSI compliance. 


\subsection{REFERENCES}

Bernard, J. W. 1988. "MAP/TOP Installation Database." In Proceedings of the Enterprise Conference. Society of Manufacturing Engineers, June 5-9, 1988.

Federal Information Processing Standard Publication 146. August 24, 1988. "Govermment Open System Interconnection Profile (GOSIP)." U.S. Department of Commerce/National Bureau of Standards.

Government Computing News. 1989. "Novell Adopting NetWare in Preparation for GOSIP." Government Computing News 8(22).

Hancock, B. 1989. "Put More Fiber in Your Network." DEC Professional 36-47.

Russel, R. H. 1988. "Industrial Data Communications: MAP and Beyond." In Proceedings of the Enterprise Conference, Society of Manufacturing Engineers, June 5-9, 1988. 

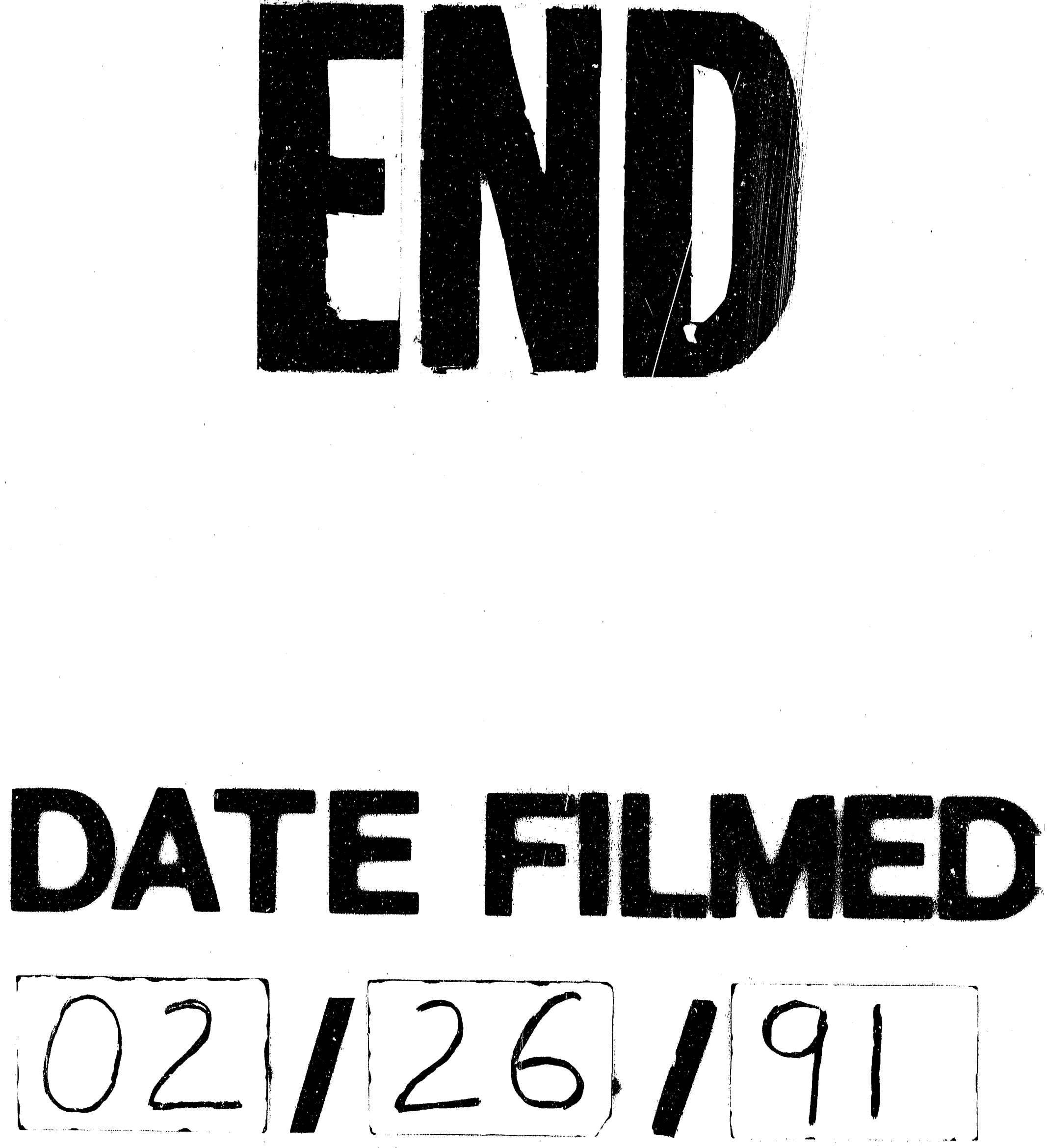
